TRANSACTIONS OF THE

AMERICAN MATHEMATICAL SOCIETY

Volume 355, Number 9, Pages 3485-3512

S 0002-9947(03)03304-X

Article electronically published on April 25, 2003

\title{
IRREDUCIBILITY OF EQUISINGULAR FAMILIES OF CURVES
}

\author{
THOMAS KEILEN
}

\begin{abstract}
In 1985 Joe Harris proved the long-standing claim of Severi that equisingular families of plane nodal curves are irreducible whenever they are nonempty. For families with more complicated singularities this is no longer true. Given a divisor $D$ on a smooth projective surface $\Sigma$ it thus makes sense to look for conditions which ensure that the family $V_{|D|}^{\text {irr }}\left(\mathcal{S}_{1}, \ldots, \mathcal{S}_{r}\right)$ of irreducible curves in the linear system $|D|_{l}$ with precisely $r$ singular points of types $\mathcal{S}_{1}, \ldots, \mathcal{S}_{r}$ is irreducible. Considering different surfaces, including general surfaces in $\mathbb{P}_{\mathbb{C}}^{3}$ and products of curves, we produce a sufficient condition of the type$$
\sum_{i=1}^{r} \operatorname{deg}\left(X\left(\mathcal{S}_{i}\right)\right)^{2}<\gamma \cdot\left(D-K_{\Sigma}\right)^{2}
$$

where $\gamma$ is some constant and $X\left(\mathcal{S}_{i}\right)$ some zero-dimensional scheme associated to the singularity type. Our results carry the same asymptotics as the best known results in this direction in the plane case, even though the coefficient is worse. For most of the surfaces considered these are the only known results in that direction.
\end{abstract}

\section{INTRODUCTION}

Equisingular families of curves have been studied quite intensively since the last century. If we fix a linear system $|D|_{l}$ on a smooth projective surface $\Sigma$ and singularity types $\mathcal{S}_{1}, \ldots, \mathcal{S}_{r}$, then we denote by $V^{i r r}=V_{|D|}^{i r r}\left(\mathcal{S}_{1}, \ldots, \mathcal{S}_{r}\right)$ the variety of irreducible curves in $|D|_{l}$ with precisely $r$ singular points of the given types. The main questions are whether the equisingular family $V^{i r r}$ is nonempty, smooth of the expected dimension, and irreducible. For results in the plane case we refer to GLS98c, GLS00, and results on the first and the second question on other surfaces may be found in [GLS97], GLS98a], CC99], [Fla01, [Che01], [KT02]. In this paper, for the first time, the question of the irreducibility of $V^{i r r}$ for a wider range of surfaces is studied. As already families of cuspidal curves in the plane (cf. Zar35]) or nodal curves on surfaces in $\mathbb{P}_{\mathbb{C}}^{3}$ (cf. [CC99]) show, in general we cannot expect a complete answer as for families of plane nodal curves, saying that the family is irreducible whenever it is nonempty. All we may hope for are numerical conditions depending on invariants of the singularity types, the surface and the linear system, which ensure the irreducibility of $V^{i r r}$.

Received by the editors August 10, 2001 and, in revised form, February 5, 2002.

2000 Mathematics Subject Classification. Primary 14H10, 14H15, 14H20; Secondary 14J26, 14J27, 14J28, 14J70.

Key words and phrases. Algebraic geometry, singularity theory.

The author was partially supported by the DFG-Schwerpunkt "Globale Methoden in der komplexen Geometrie". The author would like to thank the referee for pointing out Example 2.5 . 
The main condition which we get (cf. Section 2) looks like

$$
\sum_{i=1}^{r} \operatorname{deg}\left(X\left(\mathcal{S}_{i}\right)\right)^{2}<\gamma \cdot\left(D-K_{\Sigma}\right)^{2}
$$

where $\gamma$ is some constant. Applying the estimates (1.6) for $\operatorname{deg}\left(X\left(\mathcal{S}_{i}\right)\right)$ from Subsection 1.3 we could replace (1.1) by

$$
\sum_{i=1}^{r} \tau\left(\mathcal{S}_{i}\right)^{2}<\frac{\gamma}{9} \cdot\left(D-K_{\Sigma}\right)^{2}
$$

in the case of analytical types, and in the topological case by

$$
\sum_{i=1}^{r}\left(\mu\left(\mathcal{S}_{i}\right)+\frac{4}{3}\right)^{2}<\frac{4 \cdot \gamma}{9} \cdot\left(D-K_{\Sigma}\right)^{2}
$$

In this section we introduce the basic concepts and notation used throughout the paper, and we state several important known facts. Section 2 contains the main results and their proofs, omitting the technical details. These are presented in Section 3 and Section 4

1.1. General Assumptions and Notation. Throughout this article $\Sigma$ will denote a smooth projective surface over $\mathbb{C} ; \mathbb{N}$ denotes the set of nonnegative integers.

We will denote by $\operatorname{Div}(\Sigma)$ the group of divisors on $\Sigma$ and by $K_{\Sigma}$ its canonical divisor. If $D$ is any divisor on $\Sigma, \mathcal{O}_{\Sigma}(D)$ shall be the corresponding invertible sheaf, and we will sometimes write $H^{\nu}(X, D)$ instead of $H^{\nu}\left(X, \mathcal{O}_{X}(D)\right)$. A curve $C \subset \Sigma$ will be an effective (nonzero) divisor, that is, a one-dimensional locally principal scheme, not necessarily reduced; however, an irreducible curve shall be reduced by definition. $|D|_{l}$ denotes the system of curves linearly equivalent to $D$. We will use the notation $\operatorname{Pic}(\Sigma)$ for the Picard group of $\Sigma$, that is, $\operatorname{Div}(\Sigma)$ modulo linear equivalence (denoted by $\sim_{l}$ ), and $\operatorname{NS}(\Sigma)$ for the Néron-Severi group, that is, $\operatorname{Div}(\Sigma)$ modulo algebraic equivalence (denoted by $\sim_{a}$ ). Given a reduced curve $C \subset \Sigma$, we will write $g(C)$ for its geometric genus.

Given any closed subscheme $X$ of a scheme $Y$, we denote by $\mathcal{J}_{X}=\mathcal{J}_{X / Y}$ the ideal sheaf of $X$ in $\mathcal{O}_{Y}$. If $X$ is zero-dimensional, we denote by $\# X$ the number of points in its support $\operatorname{supp}(X)$ and by $\operatorname{deg}(X)=\sum_{z \in Y} \operatorname{dim}_{\mathbb{C}}\left(\mathcal{O}_{Y, z} / \mathcal{J}_{X / Y, z}\right)$ its degree.

If $X \subset \Sigma$ is a zero-dimensional scheme on $\Sigma$ and $D \in \operatorname{Div}(\Sigma)$, we denote by $\left|\mathcal{J}_{X / \Sigma}(D)\right|_{l}$ the linear system of curves $C$ in $|D|_{l}$ with $X \subset C$.

If $L \subset \Sigma$ is any reduced curve and $X \subset \Sigma$ a zero-dimensional scheme, we define the residue scheme $X: L \subset \Sigma$ of $X$ by the ideal sheaf $\mathcal{J}_{X: L / \Sigma}=\mathcal{J}_{X / \Sigma}: \mathcal{J}_{L / \Sigma}$ with stalks

$$
\mathcal{J}_{X: L / \Sigma, z}=\mathcal{J}_{X / \Sigma, z}: \mathcal{J}_{L / \Sigma, z}
$$

where ":" denotes the ideal quotient. This leads to the definition of the trace scheme $X \cap L \subset L$ of $X$ via the ideal sheaf $\mathcal{J}_{X \cap L / L}$ given by the exact sequence

$$
0 \longrightarrow \mathcal{J}_{X: L / \Sigma}(-L) \stackrel{\cdot L}{\longrightarrow} \mathcal{J}_{X / \Sigma} \longrightarrow \mathcal{J}_{X \cap L / L} \longrightarrow 0
$$


1.2. Singularity Types. The germ $(C, z) \subset(\Sigma, z)$ of a reduced curve $C \subset \Sigma$ at a point $z \in \Sigma$ is called a plane curve singularity, and two plane curve singularities $(C, z)$ and $\left(C^{\prime}, z^{\prime}\right)$ are said to be topologically (respectively, analytically equivalent) if there is a homeomorphism (respectively, an analytical isomorphism) $\Phi:(\Sigma, z) \rightarrow\left(\Sigma, z^{\prime}\right)$ such that $\Phi(C)=C^{\prime}$. We call an equivalence class with respect to these equivalence relations a topological (respectively, analytical) singularity type. The following are known to be invariants of the topological type $\mathcal{S}$ of the plane curve singularity $(C, z): r(\mathcal{S})=r(C, z)$, the number of branches of $(C, z) ; \tau^{e s}(\mathcal{S})=\tau^{e s}(C, z)$, the codimension of the $\mu$-constant stratum in the semiuniversal deformation of $(C, z) ; \delta(\mathcal{S})=\delta(C, z)=\operatorname{dim}_{\mathbb{C}}\left(\nu_{*} \mathcal{O}_{\widetilde{C}, z} / \mathcal{O}_{C, z}\right)$,. the delta invariant of $\mathcal{S}$, where $\nu:(\widetilde{C}, z) \rightarrow(C, z)$ is a normalisation of $(C, z)$; and $\mu(\mathcal{S})=\mu(C, z)=\operatorname{dim}_{\mathbb{C}} \mathcal{O}_{\Sigma, z} /\left(\frac{\partial f}{\partial x}, \frac{\partial f}{\partial y}\right)$, the Milnor number of $\mathcal{S}$, where $f \in \mathcal{O}_{\Sigma, z}$ denotes a local equation of $(C, z)$ with respect to the local coordinates $x$ and $y$. For the analytical type $\mathcal{S}$ of $(C, z)$ we have as additional invariant, the Tyurina number of $\mathcal{S}$, defined as $\tau(\mathcal{S})=\tau(C, z)=\operatorname{dim}_{\mathbb{C}} \mathcal{O}_{\Sigma, z} /\left(f, \frac{\partial f}{\partial x}, \frac{\partial f}{\partial y}\right)$. We recall the relation $2 \delta(\mathcal{S})=\mu(\mathcal{S})+r(\mathcal{S})-1$ (cf. [Mil68, Chapter 10]). Furthermore, since the $\delta$-constant stratum of the semiuniversal deformation of $(C, z)$ contains the $\mu$-constant stratum and since its codimension is just $\delta(\mathcal{S})$, we have $\delta(\mathcal{S}) \leq \tau^{e s}(\mathcal{S})$ (see also DH88); hence

$$
\mu(\mathcal{S}) \leq 2 \delta(\mathcal{S}) \leq 2 \tau^{e s}(\mathcal{S})
$$

1.3. Singularity Schemes. For a reduced curve $C \subset \Sigma$ we recall the definition of the zero-dimensional schemes $X^{e s}(C) \subseteq X^{s}(C)$ and $X^{e a}(C) \subseteq X^{a}(C)$ from GLS00. They are defined by the ideal sheaves $\mathcal{J}_{X^{e s}(C) / \Sigma}, \mathcal{J}_{X^{s}(C) / \Sigma}, \mathcal{J}_{X^{e a}(C) / \Sigma}$, and $\mathcal{J}_{X^{a}(C) / \Sigma}$, respectively, given by the following stalks:

- $\mathcal{J}_{X{ }^{e s}(C) / \Sigma, z}=I^{e s}(C, z)=\left\{g \in \mathcal{O}_{\Sigma, z} \mid f+\varepsilon g\right.$ is equisingular over $\left.\mathbb{C}[\varepsilon] /\left(\varepsilon^{2}\right)\right\}$, where $f \in \mathcal{O}_{\Sigma, z}$ is a local equation of $C$ at $z . I^{e s}(C, z)$ is called the equisingularity ideal of $(C, z)$.

- $\mathcal{J}_{X^{s}(C) / \Sigma, z}=\left\{g \in \mathcal{O}_{\Sigma, z} \mid g\right.$ goes through the cluster $\left.\mathcal{C} \ell\left(C, T^{*}(C, z)\right)\right\}$, where $T^{*}(C, z)$ denotes the essential subtree of the complete embedded resolution tree of $(C, z)$.

- $\mathcal{J}_{X}{ }^{e a}(C) / \Sigma, z=I^{e a}(C, z)=\left(f, \frac{\partial f}{\partial x}, \frac{\partial f}{\partial y}\right) \subseteq \mathcal{O}_{\Sigma, z}$, where $x, y$ denote local coordinates of $\Sigma$ at $z$ and $f \in \mathcal{O}_{\Sigma, z}$ is a local equation of $C$. $I^{e a}(C, z)$ is called the Tyurina ideal of $(C, z)$.

- $\mathcal{J}_{X^{a}(C) / \Sigma, z}=I^{a}(C, z) \subseteq \mathcal{O}_{\Sigma, z}$, where we refer for the somewhat lengthy definition of $I^{a}(C, z)$ to GLS00, Section 1.3].

We call $X^{e s}(C)$ the equisingularity scheme of $C$ and $X^{s}(C)$ its singularity scheme. Analogously we call $X^{e a}(C)$ the equianalytical singularity scheme of $C$ and $X^{a}(C)$ its analytical singularity scheme.

Throughout this article we will frequently treat topological and analytical singularities at the same time. Whenever we do so, we will write $X^{*}(C)$ for $X^{e s}(C)$, respectively for $X^{e a}(C)$, and similarly $X(C)$ for $X^{s}(C)$, respectively for $X^{a}(C)$. 
In [Los98, Propositions 2.19 and 2.20 and in Remarks 2.40 (see also [GLS00]) and 2.41 , it is shown that, fixing a point $z \in \Sigma$ and a topological (respectively, analytical) type $\mathcal{S}$, the singularity schemes (respectively, analytical singularity schemes) having the same topological (respectively, analytical) type are parametrised by an irreducible Hilbert scheme, which we are going to denote by $\operatorname{Hilb}_{z}(\mathcal{S})$. This then leads to an irreducible family

$$
\operatorname{Hilb}(\mathcal{S})=\coprod_{z \in \Sigma} \operatorname{Hilb}_{z}(\mathcal{S})
$$

In particular, equisingular (respectively, equianalytical) singularities have singularity schemes (respectively, analytical singularity schemes) of the same degree (see also [GLS98c or Los98], Lemma 2.8). The same is of course true regarding the equisingularity scheme (respectively, the equianalytical singularity scheme). If $C \subset \Sigma$ is a reduced curve such that $z$ is a singular point of topological (respectively, analytical) type $\mathcal{S}$, we may therefore define $\operatorname{deg}(X(\mathcal{S}))=\operatorname{deg}(X(C), z)$ and $\operatorname{deg}\left(X^{*}(\mathcal{S})\right)=\operatorname{deg}\left(X^{*}(C), z\right)$. We note that, with this notation, $\operatorname{dim}_{\operatorname{Hilb}}(\mathcal{S})=$ $\operatorname{deg}(X(\mathcal{S}))-\operatorname{deg}\left(X^{*}(\mathcal{S})\right)-2$ for any $z \in \Sigma$, and thus

$$
\operatorname{dim} \operatorname{Hilb}(\mathcal{S})=\operatorname{deg}(X(\mathcal{S}))-\operatorname{deg}\left(X^{*}(\mathcal{S})\right) .
$$

In applications it is convenient to replace the degree of an (analytical) singularity scheme by an upper bound in known invariants of the singularities. From [Los98, p. 28 , p. 103 , and Lemma 2.44 , it follows that for a topological (respectively, analytical) singularity type $\mathcal{S}$ one has

$$
\operatorname{deg}\left(X^{a}(\mathcal{S})\right) \leq 3 \tau(\mathcal{S}) \quad \text { and } \quad \operatorname{deg}\left(X^{s}(\mathcal{S})\right) \leq \frac{3}{2} \mu(\mathcal{S})+2 .
$$

1.4. Equisingular Families. Given a divisor $D \in \operatorname{Div}(\Sigma)$ and topological or analytical singularity types $\mathcal{S}_{1}, \ldots, \mathcal{S}_{r}$, we denote by $V=V_{|D|}\left(\mathcal{S}_{1}, \ldots, \mathcal{S}_{r}\right)$ the locally closed subspace of $|D|_{l}$ of reduced curves in the linear system $|D|_{l}$ having precisely $r$ singular points of types $\mathcal{S}_{1}, \ldots, \mathcal{S}_{r}$. By $V^{r e g}=V_{|D|}^{r e g}\left(\mathcal{S}_{1}, \ldots, \mathcal{S}_{r}\right)$ we denote the open (cf. the proof of Theorem 3.1) subset

$$
V^{\text {reg }}=\left\{C \in V \mid h^{1}\left(\Sigma, \mathcal{J}_{X(C) / \Sigma}(D)\right)=0\right\} \subseteq V .
$$

Similarly, we use the notation $V^{i r r}=V_{|D|}^{i r r}\left(\mathcal{S}_{1}, \ldots, \mathcal{S}_{r}\right)$ to denote the open subset of irreducible curves in the space $V$, and we set $V^{\text {irr,reg }}=V_{|D|}^{\text {irr,reg }}\left(\mathcal{S}_{1}, \ldots, \mathcal{S}_{r}\right)=$ $V^{i r r} \cap V^{r e g}$, which is open in $V^{r e g}$ and in $V$. If a type $\mathcal{S}$ occurs $k>1$ times, we rather write $k \mathcal{S}$ than $\mathcal{S}, . k ., \mathcal{S}$. We call these families of curves equisingular families of curves.

We say that $V$ is $T$-smooth at $C \in V$ if the germ $(V, C)$ is smooth of the (expected) dimension $\operatorname{dim}|D|_{l}-\operatorname{deg}\left(X^{*}(C)\right)$.

By Los98, Proposition 2.1 (see also GK89, GL96, GLS00]) T-smoothness of $V$ at $C$ follows from the vanishing of $H^{1}\left(\Sigma, \mathcal{J}_{X^{*}(C) / \Sigma}(C)\right)$, since the tangent space of $V$ at $C$ may be identified with $H^{0}\left(\Sigma, \mathcal{J}_{X^{*}(C) / \Sigma}(C)\right) / H^{0}\left(\Sigma, \mathcal{O}_{\Sigma}\right)$.

\footnotetext{
${ }^{1} V^{\text {reg }}$ should not be confused with $\left\{C \in V \mid h^{1}\left(\Sigma, \mathcal{J}_{X^{*}(C) / \Sigma}(D)\right)=0\right\}$, which is the part of $V$ where $V$ is smooth of the expected dimension. Curves in the latter subscheme are often called regular (c.f. [CC99]). See also Example 2.5.
} 
1.5. Fibrations. Let $D \in \operatorname{Div}(\Sigma)$ be a divisor, $\mathcal{S}_{1}, \ldots, \mathcal{S}_{r}$ distinct topological or analytical singularity types, and $k_{1}, \ldots, k_{r} \in \mathbb{N} \backslash\{0\}$. We denote by $\widetilde{B}$ the irreducible parameter space

$$
\widetilde{B}=\widetilde{B}\left(k_{1} \mathcal{S}_{1}, \ldots, k_{r} \mathcal{S}_{r}\right)=\prod_{i=1}^{r} \operatorname{Sym}^{k_{i}}\left(\operatorname{Hilb}\left(\mathcal{S}_{i}\right)\right)
$$

and by $B=B\left(k_{1} \mathcal{S}_{1}, \ldots, k_{r} \mathcal{S}_{r}\right)$ the nonempty, open, irreducible and dense subspace

$$
\begin{array}{r}
B=\left\{\left(\left[X_{1,1}, \ldots, X_{1, k_{1}}\right], \ldots,\left[X_{r, 1}, \ldots, X_{r, k_{r}}\right]\right) \in \widetilde{B} \mid \operatorname{supp}\left(X_{i, j}\right) \cap \operatorname{supp}\left(X_{s, t}\right)=\emptyset\right. \\
\left.\forall 1 \leq i, s \leq r, 1 \leq j \leq k_{i}, 1 \leq t \leq k_{s}\right\} .
\end{array}
$$

Note that $\operatorname{dim}(B)$ does not depend on $\Sigma$; more precisely, with the notation of Subsection 1.3 we have

$$
\operatorname{dim}(B)=\sum_{i=1}^{r} k_{i} \cdot\left(\operatorname{deg}\left(X\left(\mathcal{S}_{i}\right)\right)-\operatorname{deg}\left(X^{*}\left(\mathcal{S}_{i}\right)\right)\right)
$$

Let us set $n=\sum_{i=1}^{r} k_{i} \operatorname{deg}\left(X\left(\mathcal{S}_{i}\right)\right)$. We then define an injective morphism

$$
\begin{aligned}
& \psi=\psi\left(k_{1} \mathcal{S}_{1}, \ldots, k_{r} \mathcal{S}_{r}\right): B\left(k_{1} \mathcal{S}_{1}, \ldots, k_{r} \mathcal{S}_{r}\right) \longrightarrow \text { Hilb }_{\Sigma}^{n} \\
& \left(\left[X_{1,1}, \ldots, X_{1, k_{1}}\right], \ldots,\left[X_{r, 1}, \ldots, X_{r, k_{r}}\right]\right) \longmapsto \bigcup_{i=1}^{r} \bigcup_{j=1}^{k_{i}} X_{i, j}
\end{aligned}
$$

where $\operatorname{Hilb}_{\Sigma}^{n}$ denotes the smooth connected Hilbert scheme of zero-dimensional schemes of degree $n$ on $\Sigma$ (cf. [Los98], Section 1.3.1).

We denote by $\Psi=\Psi_{D}\left(k_{1} \mathcal{S}_{1}, \ldots, k_{r} \mathcal{S}_{r}\right)$ the fibration of $V_{|D|}\left(k_{1} \mathcal{S}_{1}, \ldots, k_{r} \mathcal{S}_{r}\right)$ induced by $B\left(k_{1} \mathcal{S}_{1}, \ldots, k_{r} \mathcal{S}_{r}\right)$; in other words, the morphism $\Psi$ is given by

$$
\begin{aligned}
\Psi: V_{|D|}\left(k_{1} \mathcal{S}_{1}, \ldots, k_{r} \mathcal{S}_{r}\right) & \longrightarrow B\left(k_{1} \mathcal{S}_{1}, \ldots, k_{r} \mathcal{S}_{r}\right), \\
C & \longmapsto\left(\left[X_{1,1}, \ldots, X_{1, k_{1}}\right], \ldots,\left[X_{r, 1}, \ldots, X_{r, k_{r}}\right]\right),
\end{aligned}
$$

where $\operatorname{Sing}(C)=\left\{z_{i, j} \mid i=1, \ldots, r, j=1, \ldots, k_{i}\right\}, X_{i, j}=X\left(C, z_{i, j}\right)$ and $\left(C, z_{i, j}\right) \cong$ $\mathcal{S}_{i}$ for all $i=1, \ldots, r, j=1, \ldots, k_{i}$.

With the notation of Subsection 1.4 note that for $C \in V$ the fibre $\Psi^{-1}(\Psi(C))$ is the open dense subset of the linear system $\left|\mathcal{J}_{X(C) / \Sigma}(D)\right|_{l}$ consisting of the curves $C^{\prime}$ with $X\left(C^{\prime}\right)=X(C)$. In particular, the fibres of $\Psi$ restricted to $V^{r e g}$ are irreducible, and since for $C \in V^{r e g}$ the cohomology group $H^{1}\left(\Sigma, \mathcal{J}_{X(C) / \Sigma}(D)\right)$ vanishes, they are equidimensional of dimension

$$
h^{0}\left(\Sigma, \mathcal{J}_{X(C) / \Sigma}(D)\right)-1=h^{0}\left(\Sigma, \mathcal{O}_{\Sigma}(D)\right)-\sum_{i=1}^{r} k_{i} \operatorname{deg}\left(X\left(\mathcal{S}_{i}\right)\right)-1
$$

\section{The Main Results}

In this section we give sufficient conditions for the irreducibility of equisingular families of curves on certain surfaces with Picard number one, including the projective plane, general surfaces in $\mathbb{P}_{\mathbb{C}}^{3^{3}}$ and general K3-surfaces, on products of curves, and on a subclass of geometrically ruled surfaces. 


\subsection{Surfaces with Picard Number One.}

Theorem 2.1. Let $\Sigma$ be a surface such that

(i) $\mathrm{NS}(\Sigma)=L \cdot \mathbb{Z}$ with $L$ ample, and

(ii) $h^{1}(\Sigma, C)=0$, whenever $C$ is effective.

Let $D \in \operatorname{Div}(\Sigma)$, let $\mathcal{S}_{1}, \ldots, \mathcal{S}_{r}$ be pairwise distinct topological or analytical singularity types and let $k_{1}, \ldots, k_{r} \in \mathbb{N} \backslash\{0\}$.

Suppose that

$$
\begin{aligned}
& D-K_{\Sigma} \text { is big and nef, } \\
& D+K_{\Sigma} \text { is nef, } \\
& \sum_{i=1}^{r} k_{i} \operatorname{deg}\left(X\left(\mathcal{S}_{i}\right)\right)<\beta \cdot\left(D-K_{\Sigma}\right)^{2} \quad \text { for some } 0<\beta \leq \frac{1}{4} \text {, and } \\
& \sum_{i=1}^{r} k_{i} \operatorname{deg}\left(X\left(\mathcal{S}_{i}\right)\right)^{2}<\gamma \cdot\left(D-K_{\Sigma}\right)^{2} \text {, where } \\
& \quad \gamma=\frac{(1+\sqrt{1-4 \beta})^{2} \cdot L^{2}}{4 \cdot \chi\left(\mathcal{O}_{\Sigma}\right)+\max \left\{0,2 \cdot K_{\Sigma} \cdot L\right\}+6 \cdot L^{2}} .
\end{aligned}
$$

Then either $V_{|D|}^{i r r}\left(k_{1} \mathcal{S}_{1}, \ldots, k_{r} \mathcal{S}_{r}\right)$ is empty or it is irreducible of the expected dimension.

Remark 2.2. If we set

$$
\gamma=\frac{36 \alpha}{(3 \alpha+4)^{2}} \quad \text { with } \quad \alpha=\frac{4 \cdot \chi\left(\mathcal{O}_{\Sigma}\right)+\max \left\{0,2 \cdot K_{\Sigma} \cdot L\right\}+6 \cdot L^{2}}{L^{2}},
$$

then a simple calculation shows that (2.3) becomes redundant. For this we have to take into account that $\operatorname{deg}(X(\mathcal{S})) \geq 3$ for any singularity type $\mathcal{S}$. The claim then follows with $\beta=\frac{1}{3} \cdot \gamma \leq \frac{1}{4}$.

We now apply the result in several special cases.

Corollary 2.3. Let $d \geq 3$, let $L \subset \mathbb{P}_{\mathbb{C}}^{3^{2}}$ be a line, and let $\mathcal{S}_{1}, \ldots, \mathcal{S}_{r}$ be topological or analytical singularity types.

Suppose that

$$
\sum_{i=1}^{r} \operatorname{deg}\left(X\left(\mathcal{S}_{i}\right)\right)^{2}<\frac{90}{289} \cdot(d+3)^{2} .
$$

Then either $V_{|d L|}^{i r r}\left(\mathcal{S}_{1}, \ldots, \mathcal{S}_{r}\right)$ is empty or it is irreducible and T-smooth.

Many authors were concerned with the question in the case of plane curves with nodes and cusps or with nodes and one more complicated singularity or simply with ordinary multiple points; cf. e.g. Sev21, AC83, Har85, Kan89a, Kan89b, Ran89], Shu91b, Shu91a], [Bar93, [Shu94], Shu96b, Shu96a, Wal96], Los98, GLS98a, GLS98b, Bru99, GLS00. Using techniques particularly designed for these cases they get of course better results than we may expect to.

The best general results in this case can be found in [GLS00] (see also [Los98], Corollary 6.1). Given a plane curve of degree $d$, omitting nodes and cusps, they get

$$
\sum_{i=1}^{r}\left(\tau^{*}\left(\mathcal{S}_{i}\right)+2\right)^{2} \leq \frac{9}{10} \cdot d^{2}
$$


as the main irreducibility condition, where $\tau^{*}\left(\mathcal{S}_{i}\right)=\tau\left(\mathcal{S}_{i}\right)$ in the analytical case (respectively, $\tau^{*}\left(\mathcal{S}_{i}\right)=\tau^{e s}\left(\mathcal{S}_{i}\right)$ in the topological case). By Subsection 1.2 we know that $\mu\left(\mathcal{S}_{i}\right) \leq 2 \cdot \tau^{e s}\left(\mathcal{S}_{i}\right)$. Thus, in view of (1.2), (1.3), (1.4) and of Theorem 2.1]we get the sufficient condition

$$
\sum_{i=1}^{r}\left(\tau^{*}\left(\mathcal{S}_{i}\right)+\frac{2}{3}\right)^{2}<\frac{10}{289} \cdot(d+3)^{2},
$$

which has the same asymptotics. However, the coefficients differ by a factor of about 26 .

A smooth complete intersection surface with Picard number one satisfies the assumptions of Theorem 2.1] Thus by the Noether Theorem of the result applies in particular to general surfaces in $\mathbb{P}_{\mathbb{C}}^{3^{3}}$.

Corollary 2.4. Let $\Sigma \subset \mathbb{P}_{\mathbb{C}}^{3^{3}}$ be a smooth hypersurface of degree $n \geq 4$, let $H \subset \Sigma$ be a hyperplane section, and suppose that the Picard number of $\Sigma$ is one. Let $d>n-4$ and let $\mathcal{S}_{1}, \ldots, \mathcal{S}_{r}$ be topological or analytical singularity types.

Suppose that

$$
\sum_{i=1}^{r} \operatorname{deg}\left(X\left(\mathcal{S}_{i}\right)\right)^{2}<\frac{6 \cdot\left(n^{3}-3 n^{2}+8 n-6\right) \cdot n^{2}}{\left(n^{3}-3 n^{2}+10 n-6\right)^{2}} \cdot(d+4-n)^{2} .
$$

Then either $V_{|d H|}^{i r r}\left(\mathcal{S}_{1}, \ldots, \mathcal{S}_{r}\right)$ is empty or it is irreducible of the expected dimension.

We would like to thank the referee for pointing out the following example of reducible families $V_{|H|}^{i r r}\left(3 A_{1}\right)$ of nodal curves on surfaces in $\mathbb{P}_{\mathbb{C}}^{3^{3}}$.

Example 2.5. If $\Sigma \subset \mathbb{P}_{\mathbb{C}}^{3^{3}}$ is a general surface of degree $n \geq 4$, then there is a finite number $N>1$ of 3 -tangent planes to $\Sigma$. However, every 3 -tangent plane cuts out an irreducible 3-nodal curve on $\Sigma$, and since the Picard group is generated by a hyperplane section $H$, every 3 -nodal curve is of this form. Therefore, $V_{|H|}^{i r r}\left(3 A_{1}\right)$ consists of $N$ distinct points. It is thus reducible, but smooth of the expected dimension

$$
\operatorname{dim}\left(V_{|H|}^{i r r}\left(3 A_{1}\right)\right)=\operatorname{dim}|H|_{l}-3=0 .
$$

Note that in this situation for $C \in V_{|H|}^{i r r}\left(3 A_{1}\right)$ and $z \in \operatorname{Sing}(C)$ we have $\mathcal{J}_{X(C) / \Sigma, z}=$ $\mathfrak{m}_{\Sigma, z}^{2}$ and thus

$$
h^{1}\left(\Sigma, \mathcal{J}_{X(C) / \Sigma}(H)\right)=6>0 .
$$

Therefore, $V_{|H|}^{\text {irr, reg }}\left(3 A_{1}\right)=\emptyset$. The parameter space $B$ is just $\operatorname{Sym}^{3}(\Sigma)$.

A general K3-surface has Picard number one and in this situation, by the Kodaira Vanishing Theorem, $\Sigma$ also satisfies the assumption (ii) in Theorem [2.1]

Corollary 2.6. Let $\Sigma$ be a smooth K3-surface with $\mathrm{NS}(\Sigma)=L \cdot \mathbb{Z}$ with L ample and set $n=L^{2}$. Let $d>0, D \sim_{a} d L$ and let $\mathcal{S}_{1}, \ldots, \mathcal{S}_{r}$ be topological or analytical singularity types.

Suppose that

$$
\sum_{i=1}^{r} \operatorname{deg}\left(X\left(\mathcal{S}_{i}\right)\right)^{2}<\frac{54 n^{2}+72 n}{(11 n+12)^{2}} \cdot d^{2} \cdot n .
$$

Then either $V_{|D|}^{i r r}\left(\mathcal{S}_{1}, \ldots, \mathcal{S}_{r}\right)$ is empty or it is irreducible of the expected dimension. 
2.2. Products of Curves. If $\Sigma=C_{1} \times C_{2}$ is the product of two smooth projective curves, then for a general choice of $C_{1}$ and $C_{2}$ the Néron-Severi group will be generated by two fibres of the canonical projections, by abuse of notation also denoted by $C_{1}$ and $C_{2}$. If both curves are elliptic, then "general" just means that the two curves are non-isogenous.

Theorem 2.7. Let $C_{1}$ and $C_{2}$ be two smooth projective curves of genera $g_{1}$ and $g_{2}$, respectively, with $g_{1} \geq g_{2} \geq 0$, such that for $\Sigma=C_{1} \times C_{2}$ the Néron-Severi group is $\mathrm{NS}(\Sigma)=C_{1} \mathbb{Z} \oplus C_{2} \mathbb{Z}$.

Let $D \in \operatorname{Div}(\Sigma)$ such that $D \sim_{a} a C_{1}+b C_{2}$ with $a>\max \left\{2 g_{2}-2,2-2 g_{2}\right\}$ and $b>\max \left\{2 g_{1}-2,2-2 g_{1}\right\}$, let $\mathcal{S}_{1}, \ldots, \mathcal{S}_{r}$ be pairwise distinct topological or analytical singularity types and $k_{1}, \ldots, k_{r} \in \mathbb{N} \backslash\{0\}$.

Suppose that

$$
\sum_{i=1}^{r} k_{i} \operatorname{deg}\left(X\left(\mathcal{S}_{i}\right)\right)^{2}<\gamma \cdot\left(D-K_{\Sigma}\right)^{2}
$$

where $\gamma$ may be taken from the following table with $\alpha=\frac{a-2 g_{2}+2}{b-2 g_{1}+2}>0$.

\begin{tabular}{|c|c|c|c|}
\hline$g_{1}$ & $g_{2}$ & & $\gamma$ \\
\hline 0 & 0 & & $\frac{1}{24}$ \\
\hline 1 & 0 & & $\frac{1}{\max \{322 \alpha\}}$ \\
\hline$\geq 2$ & 0 & & $\frac{1}{\max \left\{24+16 a_{1} 4 a_{1} \alpha\right\}}$ \\
\hline 1 & 1 & & 1 \\
\hline \multirow{2}{*}{$\geq 2$} & $\lambda$ & & $\overline{\max \left\{32,2 \alpha, \frac{2}{\alpha}\right\}}$ \\
\hline & $\geq 1$ & $\max \{$ & $24+16 g_{1}+16 g_{2}, 4 g_{1} \alpha, \frac{4 g_{2}}{\alpha}$ \\
\hline
\end{tabular}

Then either $V_{|D|}^{i r r}\left(k_{1} \mathcal{S}_{1}, \ldots, k_{r} \mathcal{S}_{r}\right)$ is empty or it is irreducible of the expected dimension.

Only in the case $\Sigma \cong \mathbb{P}_{\mathbb{C}}^{3^{1}} \times \mathbb{P}_{\mathbb{C}}^{3^{1}}$ we get a constant $\gamma$ which does not depend on the chosen divisor $D$, while in the remaining cases the ratio of $a$ and $b$ is involved in $\gamma$. This means that an asymptotical behaviour can only be examined if the ratio remains unchanged.

2.3. Geometrically Ruled Surfaces. Let $\pi: \Sigma=\mathbb{P}(\mathcal{E}) \rightarrow C$ be a geometrically ruled surface with normalised bundle $\mathcal{E}$ (in the sense of [Har77], V.2.8.1). The Néron-Severi group of $\Sigma$ is $\operatorname{NS}(\Sigma)=C_{0} \mathbb{Z} \oplus F \mathbb{Z}$ with intersection matrix $\left(\begin{array}{cc}-e & 1 \\ 1 & 0\end{array}\right)$, where $F \cong \mathbb{P}_{\mathbb{C}}^{3^{1}}$ is a fibre of $\pi, C_{0}$ a section of $\pi$ with $\mathcal{O}_{\Sigma}\left(C_{0}\right) \cong \mathcal{O}_{\mathbb{P}(\mathcal{E})}(1), g=g(C)$ the genus of $C, \mathfrak{e}=\Lambda^{2} \mathcal{E}$ and $e=-\operatorname{deg}(\mathfrak{e}) \geq-g$. For the canonical divisor we have $K_{\Sigma} \sim_{a}-2 C_{0}+(2 g-2-e) \cdot F$.

Theorem 2.8. Let $\pi: \Sigma \rightarrow C$ be a geometrically ruled surface with $e \leq 0$. Let $D=a C_{0}+b F \in \operatorname{Div}(\Sigma)$ with $a \geq 2, b>2 g-2+\frac{a e}{2}$, and if $g=0$, then $b \geq 2$. Let $\mathcal{S}_{1}, \ldots, \mathcal{S}_{r}$ be pairwise distinct topological or analytical singularity types and $k_{1}, \ldots, k_{r} \in \mathbb{N} \backslash\{0\}$. 
Suppose that

$$
\sum_{i=1}^{r} k_{i} \operatorname{deg}\left(X\left(\mathcal{S}_{i}\right)\right)^{2}<\gamma \cdot\left(D-K_{\Sigma}\right)^{2}
$$

where $\gamma$ may be taken from the following table with $\alpha=\frac{a+2}{b+2-2 g-\frac{a e}{2}}>0$.

\begin{tabular}{|r|c|c|}
\hline \multicolumn{1}{|c|}{$g$} & $e$ & $\gamma$ \\
\hline 0 & 0 & $\frac{1}{24}$ \\
1 & 0 & $\frac{1}{\max \{24,2 \alpha\}}$ \\
1 & -1 & $\frac{1}{\max \left\{\min \left\{30+\frac{16}{\alpha}+4 \alpha, 40+9 \alpha\right\}, \frac{13}{2} \alpha\right\}}$ \\
$\geq 2$ & 0 & $\frac{1}{\max \{24+16 g, 4 g \alpha\}}$ \\
$\geq 2$ & $<0$ & $\frac{1}{\max \left\{\min \left\{24+16 g-9 e \alpha, 18+16 g-9 e \alpha-\frac{16}{e \alpha}\right\}, 4 g \alpha-9 e \alpha\right\}}$ \\
\hline
\end{tabular}

Then either $V_{|D|}^{i r r}\left(k_{1} \mathcal{S}_{1}, \ldots, k_{r} \mathcal{S}_{r}\right)$ is empty or it is irreducible of the expected dimension.

Once more, only in the case $g=0$, i.e., when $\Sigma \cong \mathbb{P}_{\mathbb{C}}^{3^{1}} \times \mathbb{P}_{\mathbb{C}}^{3^{1}}$, we are in the lucky situation that the constant $\gamma$ does not at all depend on the chosen divisor $D$, whereas in the case $g \geq 1$ the ratio of $a$ and $b$ is involved in $\gamma$. This means that an asymptotical behaviour can only be examined if the ratio remains unchanged.

If $\Sigma$ is a product $C \times \mathbb{P}_{\mathbb{C}}^{3^{1}}$, the constant $\gamma$ here is the same as in Section 2.2

In Ran89] and in [GLS98a the case of nodal curves on the Hirzebruch surface $\mathbb{F}_{1}$ is treated, since this is just $\mathbb{P}_{\mathbb{C}}^{3^{2}}$ blown up at one point. $\mathbb{F}_{1}$ is an example of a geometrically ruled surface with invariant $e=1>0$, a case which we so far cannot treat with our methods, due to the section with self-intersection -1 . However, it seems to be possible to extend the methods of [GLS98a] to the situation of arbitrary ruled surfaces with positive invariant $e$, at least if we restrict ourselves to singularities that are not too bad.

2.4. The Proofs. Our approach to the problem proceeds along the lines of an unpublished result of Greuel, Lossen and Shustin (cf. GLS98b), which is based on ideas of Chiantini and Ciliberto (cf. CC99]). The basic ideas are in some respect similar to the approach used in [GLS00, replacing the "Castelnuovo-function" arguments by "Bogomolov instability".

We first show that the open subscheme $V^{\text {irr,reg }}=V_{|D|}^{\text {irr,reg }}\left(k_{1} \mathcal{S}_{1}, \ldots, k_{r} \mathcal{S}_{r}\right)$ of $V^{i r r}=V_{|D|}^{i r r}\left(k_{1} \mathcal{S}_{1}, \ldots, k_{r} \mathcal{S}_{r}\right)$, and hence its closure $\overline{V^{i r r, r e g}}$ in $V^{i r r}$, is always irreducible (cf. Theorem 3.1), and then we look for criteria which ensure that the complement of $\overline{V^{\text {irr,reg }}}$ in $V^{\text {irr }}$ is empty (cf. Section 4 ). For the latter, we consider the restriction of the morphism $\Psi: V \rightarrow B$ (cf. Subsection 1.5) to an irreducible component $V^{*}$ of $V^{i r r}$ not contained in $\overline{V^{i r r, r e g}}$. From the fact that the dimension of $V^{*}$ is at least the expected dimension $\operatorname{dim}\left(V^{\text {irr, reg }}\right)$, we deduce that the codimension of $B^{*}=\Psi\left(V^{*}\right)$ in $B$ is at most $h^{1}\left(\Sigma, \mathcal{J}_{X(C) / \Sigma}(D)\right)$, where $C \in V^{*}$ (cf. Lemma 4.7). It thus suffices to find conditions that contradict this inequality, that is, we have to get our hands on $\operatorname{codim}_{B}\left(B^{*}\right)$. However, on the surfaces that 
we consider, the non-vanishing of $h^{1}\left(\Sigma, \mathcal{J}_{X(C) / \Sigma}(D)\right)$ means in some sense that the zero-dimensional scheme $X(C)$ is in a special position. We may thus hope to realise large parts $X_{i}^{0}$ of $X(C)$ on curves $\Delta_{i}$ of "small degree" $(i=1, \ldots, m)$, which would impose at least $\# X_{i}^{0}-\operatorname{dim}\left|\Delta_{i}\right|_{l}$ conditions on $X(C)$, giving rise to a lower bound $\sum_{i=1}^{m} \# X_{i}^{0}-\operatorname{dim}\left|\Delta_{i}\right|_{l}$ for $\operatorname{codim}_{B}\left(B^{*}\right)$. The $X_{i}^{0}$ 's and the $\Delta_{i}$ 's are found in Lemma 4.1 with the aid of certain Bogomolov unstable rank-two bundles. It thus finally remains (cf. Lemmata 4.3, 4.4 and 4.6) to give conditions that imply

$$
\sum_{i=1}^{m} \# X_{i}^{0}-\operatorname{dim}\left|\Delta_{i}\right|_{l}>h^{1}\left(\Sigma, \mathcal{J}_{X(C) / \Sigma}(D)\right) \text {. }
$$

These considerations lead to the following proofs.

Proof of Theorem [2.1. We may assume that $V^{\text {irr }}$ is nonempty. By Theorem 3.1 it suffices to show that $V^{i r r}=\overline{V^{i r r, r e g}}$.

Suppose the contrary, i.e., there is an irreducible curve $C_{0} \in V^{\text {irr }} \backslash \overline{V^{\text {irr,reg }}}$; in particular, $h^{1}\left(\Sigma, \mathcal{J}_{X_{0} / \Sigma}(D)\right)>0$ for $X_{0}=X\left(C_{0}\right)$. Since

$$
\operatorname{deg}\left(X_{0}\right)=\sum_{i=1}^{r} k_{i} \operatorname{deg}\left(X\left(\mathcal{S}_{i}\right)\right)
$$

and $\sum_{z \in \Sigma}\left(\operatorname{deg}\left(X_{0, z}\right)\right)^{2}=\sum_{i=1}^{r} k_{i} \operatorname{deg}\left(X\left(\mathcal{S}_{i}\right)\right)^{2}$, the assumptions (0)-(3) of Lemma 4.1 and (4) of Lemma 4.3 are fulfilled. Thus Lemma 4.3 implies that $C_{0}$ satisfies condition (4.19) in Lemma 4.7, which it cannot satisfy by the same lemma. Thus we have derived a contradiction.

Proof of Theorem 2.7. The assumptions on $a$ and $b$ ensure that $D-K_{\Sigma}$ is big and nef and that $D+K_{\Sigma}$ is nef. Thus, once we know that (2.5) implies condition (3) in Lemma 4.1 we can do the same proof as in Theorem 2.1, just replacing Lemma 4.3 by Lemma 4.4 .

For condition (3) we note that

$$
\sum_{i=1}^{r} k_{i} \operatorname{deg}\left(X\left(\mathcal{S}_{i}\right)\right) \leq \sum_{i=1}^{r} k_{i} \cdot\left(\operatorname{deg}\left(X\left(\mathcal{S}_{i}\right)\right)\right)^{2} \leq \frac{1}{24} \cdot\left(D-K_{\Sigma}\right)^{2}<\frac{1}{4} \cdot\left(D-K_{\Sigma}\right)^{2} .
$$

Proof of Theorem 2.8. The proof is identical to that of Theorem 2.7 just replacing Lemma 4.4 by Lemma 4.6 .

2.5. Some Remarks. What are the obstructions to our approach?

First, the Bogomolov instability does not give much information about the curves $\Delta_{i}$ apart from their existence and the fact that they are in some sense "small" compared with the divisor $D$. We are thus bound to the study of surfaces where we have a good knowledge of the dimension of arbitrary complete linear systems. Second, in order to derive the above inequality, many nasty calculations are necessary which strongly depend on the particular structure of the Néron-Severi group of the surface, that is, we are restricted to surfaces where the Néron-Severi group is not too large and the intersection pairing is not too hard (cf. Lemmata 4.3, 4.4 and 4.6). Finally, in order to ensure the Bogomolov instability of the vector bundle considered throughout the proof of Lemma 4.1 we heavily use the fact that the surface $\Sigma$ does not contain any curve of negative self-intersection, which excludes e.g. general Hirzebruch surfaces. 
If the number of irreducible curves of negative self-intersection is not too large, one might overcome this last obstacle with the technique used in [GLS98a]. That is, we would have to show that under certain additional conditions the singular points of the considered curves could be independently moved; in particular, they could be moved off the exceptional curves - more precisely, the subvariety of $V^{i r r}$ of curves whose singular locus does not lie on any exceptional curve is dense in $V^{i r r}$. For this, one basically just needs criteria for the existence of "small" curves realising a zero-dimensional scheme slightly bigger than the equisingularity scheme (respectively, the equianalytical singularity scheme) of the members in $V^{i r r}$. For example, in the case of curves with $r$ nodes, that means the existence of curves passing through $r$ arbitrary points and having multiplicity two in one of them.

In Section 3 we not only prove that $V^{i r r, r e g}$ is irreducible, but also that this indeed remains true if we drop the requirement that the curves should be irreducible, i.e., we show that $V^{r e g}$ is irreducible. However, unfortunately our approach does not give conditions for the emptiness of the complement of $\overline{V^{r e g}}$, and thus we cannot say anything about the irreducibility of the variety of possibly reducible curves in $|D|_{l}$ with prescribed singularities. The reason for this is that in the proof of Lemma 4.1 we use the Bézout Theorem to estimate $D \cdot \Delta_{i}$.

\section{3. $V^{\text {irr, reg }}$ IS IRREDUCIBLE}

We now show that $V^{\text {irr,reg }}$ is always irreducible. We do this by showing that under $\Psi: V \rightarrow B$ every irreducible component of $V^{i r r, r e g}$ is smooth and maps dominant to the irreducible variety $B$ with irreducible fibres.

Theorem 3.1. Let $D \in \operatorname{Div}(\Sigma), \mathcal{S}_{1}, \ldots, \mathcal{S}_{r}$ be pairwise distinct topological or analytical singularity types and $k_{1}, \ldots, k_{r} \in \mathbb{N} \backslash\{0\}$.

If $V_{|D|}^{\text {irr,reg }}\left(k_{1} \mathcal{S}_{1}, \ldots, k_{r} \mathcal{S}_{r}\right)$ is nonempty, then it is a T-smooth, irreducible, open subset of $V_{|D|}^{i r r}\left(k_{1} \mathcal{S}_{1}, \ldots, k_{r} \mathcal{S}_{r}\right)$ of dimension $\operatorname{dim}|D|_{l}-\sum_{i=1}^{r} k_{i} \operatorname{deg}\left(X^{*}\left(\mathcal{S}_{i}\right)\right)$.

Proof. Since $V_{|D|}^{i r r, r e g}\left(k_{1} \mathcal{S}_{1}, \ldots, k_{r} \mathcal{S}_{r}\right)$ is an open subset of $V_{|D|}^{r e g}\left(k_{1} \mathcal{S}_{1}, \ldots, k_{r} \mathcal{S}_{r}\right)=$ $V^{r e g}$, it suffices to show the claim for $V^{r e g}$.

Let us consider the following maps from Subsection 1.5

$$
\Psi=\Psi_{D}\left(k_{1} \mathcal{S}_{1}, \ldots, k_{r} \mathcal{S}_{r}\right): V=V_{|D|}\left(k_{1} \mathcal{S}_{1}, \ldots, k_{r} \mathcal{S}_{r}\right) \longrightarrow B\left(k_{1} \mathcal{S}_{1}, \ldots, k_{r} \mathcal{S}_{r}\right)
$$

and

$$
\psi=\psi\left(k_{1} \mathcal{S}_{1}, \ldots, k_{r} \mathcal{S}_{r}\right): B\left(k_{1} \mathcal{S}_{1}, \ldots, k_{r} \mathcal{S}_{r}\right) \longrightarrow \mathrm{Hilb}_{\Sigma}^{n}
$$

Step 1: Every irreducible component $V^{*}$ of $V^{\text {reg }}$ is T-smooth of dimension $\operatorname{dim}|D|_{l}-\sum_{i=1}^{r} k_{i} \operatorname{deg}\left(X^{*}\left(\mathcal{S}_{i}\right)\right)$. By [Los98], Proposition $2.1(\mathrm{c} 2), V^{*}$ is T-smooth at any $C \in V^{*}$ of dimension $\operatorname{dim}|D|_{l}-\operatorname{deg}\left(X^{*}(C)\right)$, since $h^{1}\left(\Sigma, \mathcal{J}_{X^{*} / \Sigma}(D)\right)=0$. Note that $\operatorname{deg}\left(X^{*}(C)\right)=\sum_{i=1}^{r} k_{i} \operatorname{deg}\left(X^{*}\left(\mathcal{S}_{i}\right)\right)$ only depends on $k_{1} \mathcal{S}_{1}, \ldots, k_{r} \mathcal{S}_{r}$ (cf. Subsection 1.3).

Step 2: $V^{r e g}$ is open in $V$. Let $C \in V^{r e g}$; then $h^{1}\left(\Sigma, \mathcal{J}_{X(C) / \Sigma}(D)\right)=0$. Thus by semicontinuity there exists an open, dense neighbourhood $U$ of $X(C)$ in $\operatorname{Hilb}_{\Sigma}^{n}$ such that $h^{1}\left(\Sigma, \mathcal{J}_{Y / \Sigma}(D)\right)=0$ for all $Y \in U$. But then $\Psi^{-1}\left(\psi^{-1}(U)\right) \subseteq V^{r e g}$ is an open neighbourhood of $C$ in $V$, and hence $V^{r e g}$ is open in $V$.

Step 3: $\Psi$ restricted to any irreducible component $V^{*}$ of $V^{r e g}$ is dominant. Let $V^{*}$ be an irreducible component of $V^{r e g}$ and let $C \in V^{*}$. Since $\Psi^{-1}(\Psi(C))$ is an 
open, dense subset of $\left|\mathcal{J}_{X(C) / \Sigma}(D)\right|_{l}$ and since $h^{1}\left(\Sigma, \mathcal{J}_{X(C) / \Sigma}(D)\right)=0$, we have

$$
\operatorname{dim} \Psi^{-1}(\Psi(C))=h^{0}\left(\Sigma, \mathcal{J}_{X(C) / \Sigma}(D)\right)-1=\operatorname{dim}|D|_{l}-\operatorname{deg}(X(C)) .
$$

By Step 1 we know the dimension of $V^{*}$ and by Subsection 1.5 we also know the dimension of $B$. Thus we conclude

$$
\begin{aligned}
\operatorname{dim} \Psi\left(V^{*}\right) & =\operatorname{dim} V^{*}-\operatorname{dim} \Psi^{-1}(\Psi(C)) \\
& =\left(\operatorname{dim}|D|_{l}-\operatorname{deg} X^{*}(C)\right)-\left(\operatorname{dim}|D|_{l}-\operatorname{deg} X(C)\right) \\
& =\operatorname{deg}(X(C))-\operatorname{deg}\left(X^{*}(C)\right)=\operatorname{dim} B .
\end{aligned}
$$

Since $B$ is irreducible, $\Psi\left(V^{*}\right)$ must be dense in $B$.

Step 4: $V^{r e g}$ is irreducible. Let $V^{*}$ and $V^{* *}$ be two irreducible components of $V^{r e g}$. Then $\Psi\left(V^{*}\right) \cap \Psi\left(V^{* *}\right) \neq \emptyset$, and thus some fibre $F$ of $\Psi$ intersects both, $V^{*}$ and $V^{* *}$. However, the fibre is irreducible and by Step 1 both $V^{*}$ and $V^{* *}$ are smooth. Thus $F$ must be completely contained in $V^{*}$ and $V^{* *}$, which implies that $V^{*}=V^{* *}$, since both are smooth of the same dimension. Thus $V^{\text {reg }}$ is irreducible.

\section{The Technical Details}

The following lemma is the heart of the proof. Given a curve $C \in|D|_{l}$, whose (analytical) singularity scheme $X_{0}=X(C)$ is special with respect to $D$ in the sense that $h^{1}\left(\Sigma, \mathcal{J}_{X_{0} / \Sigma}(D)\right)>0$, provides a "small" curve $\Delta_{1}$ through a subscheme $X_{1}^{0}$ of $X_{0}$, so that we can reduce the problem by replacing $X_{0}$ and $D$ by $X_{0}: \Delta_{1}$ and $D-\Delta_{1}$ respectively. We can of course proceed inductively as long as the new zero-dimensional scheme is again special with respect to the new divisor.

In order to find $\Delta_{1}$ we choose a subscheme $X_{1}^{0} \subseteq X_{0}$ that is minimal among those subschemes special with respect to $D$. By Grothendieck-Serre duality,

$$
H^{1}\left(\Sigma, \mathcal{J}_{X_{1}^{0} / \Sigma}(D)\right) \cong \operatorname{Ext}^{1}\left(\mathcal{J}_{X_{1}^{0} / \Sigma}\left(D-K_{\Sigma}\right), \mathcal{O}_{\Sigma}\right)
$$

and a nontrivial element of the latter group gives rise to an extension

$$
0 \rightarrow \mathcal{O}_{\Sigma} \rightarrow E_{1} \rightarrow \mathcal{J}_{X_{1}^{0} / \Sigma}\left(D-K_{\Sigma}\right) \rightarrow 0
$$

We then show that the rank-two bundle $E_{1}$ is Bogomolov unstable and deduce the existence of a divisor $\Delta_{1}^{0}$ such that

$$
H^{0}\left(\Sigma, \mathcal{J}_{X_{1}^{0} / \Sigma}\left(D-K_{\Sigma}-\Delta_{1}^{0}\right)\right) \neq 0
$$

that is, we find a curve $\Delta_{1} \in\left|\mathcal{J}_{X_{1}^{0} / \Sigma}\left(D-K_{\Sigma}-\Delta_{1}^{0}\right)\right|_{l}$.

Lemma 4.1. Let $\Sigma$ be a surface such that any curve $C \subset \Sigma$ is nef. (Assumption $(*))$

Let $D \in \operatorname{Div}(\Sigma)$ and $X_{0} \subset \Sigma$ a zero-dimensional scheme satisfying

(0) $D-K_{\Sigma}$ is big and nef, and $D+K_{\Sigma}$ is nef,

(1) $\exists C_{0} \in|D|_{l}$ irreducible: $X_{0} \subset C_{0}$,

(2) $h^{1}\left(\Sigma, \mathcal{J}_{X_{0} / \Sigma}(D)\right)>0$, and

(3) $\operatorname{deg}\left(X_{0}\right)<\beta \cdot\left(D-K_{\Sigma}\right)^{2}$ for some $0<\beta \leq \frac{1}{4}$.

Then there exist curves $\Delta_{1}, \ldots, \Delta_{m} \subset \Sigma$ and zero-dimensional locally complete intersections $X_{i}^{0} \subseteq X_{i-1} \cap \Delta_{i}$ for $i=1, \ldots, m$, where $X_{i}=X_{i-1}: \Delta_{i}$ for $i=$ $1, \ldots, m$, such that

(a) $h^{1}\left(\Sigma, \mathcal{J}_{X_{m} / \Sigma}\left(D-\sum_{i=1}^{m} \Delta_{i}\right)\right)=0$, 
and for $i=1, \ldots, m$

(b) $h^{1}\left(\Sigma, \mathcal{J}_{X_{i}^{0} / \Sigma}\left(D-\sum_{k=1}^{i-1} \Delta_{k}\right)\right)=1$,

(c) $D \cdot \Delta_{i} \geq \operatorname{deg}\left(X_{i-1} \cap \Delta_{i}\right) \geq \operatorname{deg}\left(X_{i}^{0}\right) \geq\left(D-K_{\Sigma}-\sum_{k=1}^{i} \Delta_{k}\right) \cdot \Delta_{i} \geq \Delta_{i}^{2} \geq 0$,

(d) $\left(D-K_{\Sigma}-\sum_{k=1}^{i} \Delta_{k}-\Delta_{i}\right)^{2}>0$,

(e) $\left(D-K_{\Sigma}-\sum_{k=1}^{i} \Delta_{k}-\Delta_{i}\right) \cdot H>0$ for all $H \in \operatorname{Div}(\Sigma)$ ample, and

(f) $D-K_{\Sigma}-\sum_{k=1}^{i} \Delta_{k}$ is big and nef.

Moreover, it follows that

$$
0 \leq \frac{1}{4}\left(D-K_{\Sigma}\right)^{2}-\sum_{i=1}^{m} \operatorname{deg}\left(X_{i}^{0}\right) \leq\left(\frac{1}{2}\left(D-K_{\Sigma}\right)-\sum_{i=1}^{m} \Delta_{i}\right)^{2}
$$

Proof. We are going to find the schemes $\Delta_{i}$ and $X_{i}^{0}$ recursively. Let us therefore suppose that we have already found $\Delta_{1}, \ldots, \Delta_{i-1}$ and $X_{1}^{0}, \ldots, X_{i-1}^{0}$ satisfying (b)(f), and suppose that still $h^{1}\left(\Sigma, \mathcal{J}_{X_{i-1} / \Sigma}\left(D-\sum_{k=1}^{i-1} \Delta_{k}\right)\right)>0$.

We choose minimal $X_{i}^{0} \subseteq X_{i-1}$ such that $h^{1}\left(\Sigma, \mathcal{J}_{X_{i}^{0} / \Sigma}\left(D-\sum_{k=1}^{i-1} \Delta_{k}\right)\right)>0$.

Step 1: $h^{1}\left(\Sigma, \mathcal{J}_{X_{i}^{0} / \Sigma}\left(D-\sum_{k=1}^{i-1} \Delta_{k}\right)\right)=1$, i.e., (b) is fulfilled. Suppose it was strictly larger than one. By (0), respectively (f), and by the Kawamata-Viehweg Vanishing Theorem we have $h^{1}\left(\Sigma, \mathcal{O}_{\Sigma}\left(D-\sum_{k=1}^{i-1} \Delta_{k}\right)\right)=0$.

Thus $X_{i}^{0}$ cannot be empty, that is, $\operatorname{deg}\left(X_{i}^{0}\right) \geq 1$ and we may choose a subscheme $Y \subset X_{i}^{0}$ of degree $\operatorname{deg}(Y)=\operatorname{deg}\left(X_{i}^{0}\right)-1$. The inclusion $\mathcal{J}_{X_{i}^{0}} \hookrightarrow \mathcal{J}_{Y}$ implies $h^{0}\left(\Sigma, \mathcal{J}_{X_{i}^{0} / \Sigma}\left(D-\sum_{k=1}^{i-1} \Delta_{k}\right)\right) \leq h^{0}\left(\Sigma, \mathcal{J}_{Y / \Sigma}\left(D-\sum_{k=1}^{i-1} \Delta_{k}\right)\right)$ and the structure sequences of $Y$ and $X_{i}^{0}$ thus lead to

$$
h^{1}\left(\Sigma, \mathcal{J}_{Y / \Sigma}\left(D-\sum_{k=1}^{i-1} \Delta_{k}\right)\right) \geq h^{1}\left(\Sigma, \mathcal{J}_{X_{i}^{0} / \Sigma}\left(D-\sum_{k=1}^{i-1} \Delta_{k}\right)\right)-1>0
$$

contradicting the minimality of $X_{i}^{0}$.

Step 2: $\operatorname{deg}\left(X_{i}^{0}\right) \leq \operatorname{deg}\left(X_{0}\right)-\sum_{k=1}^{i-1} \operatorname{deg}\left(X_{k-1} \cap \Delta_{k}\right)$.

The case $i=1$ follows from the fact that $X_{1}^{0} \subseteq X_{0}$, and for $i>1$ the inclusion $X_{i}^{0} \subseteq X_{i-1}=X_{i-2}: \Delta_{i-1}$ implies

$$
\operatorname{deg}\left(X_{i}^{0}\right) \leq \operatorname{deg}\left(X_{i-2}: \Delta_{i-1}\right)=\operatorname{deg}\left(X_{i-2}\right)-\operatorname{deg}\left(X_{i-2} \cap \Delta_{i-1}\right) .
$$

It thus suffices to show that

$$
\operatorname{deg}\left(X_{i-2}\right)-\operatorname{deg}\left(X_{i-2} \cap \Delta_{i-1}\right)=\operatorname{deg}\left(X_{0}\right)-\sum_{k=1}^{i-1} \operatorname{deg}\left(X_{k-1} \cap \Delta_{k}\right) .
$$

If $i=2$, there is nothing to show. Otherwise $X_{i-2}=X_{i-3}: \Delta_{i-2}$ implies

$$
\begin{aligned}
& \operatorname{deg}\left(X_{i-2}\right)-\operatorname{deg}\left(X_{i-2} \cap \Delta_{i-1}\right) \\
& =\operatorname{deg}\left(X_{i-3}: \Delta_{i-2}\right)-\operatorname{deg}\left(X_{i-2} \cap \Delta_{i-1}\right) \\
& =\operatorname{deg}\left(X_{i-3}\right)-\operatorname{deg}\left(X_{i-3} \cap \Delta_{i-2}\right)-\operatorname{deg}\left(X_{i-2} \cap \Delta_{i-1}\right)
\end{aligned}
$$

and we are done by induction.

Step 3: There exists a "suitable" locally free rank-two vector bundle $E_{i}$. 
By the Grothendieck-Serre duality we have

$$
0 \neq H^{1}\left(\Sigma, \mathcal{J}_{X_{i}^{0} / \Sigma}\left(D-\sum_{k=1}^{i-1} \Delta_{k}\right)\right) \cong \operatorname{Ext}^{1}\left(\mathcal{J}_{X_{i}^{0} / \Sigma}\left(D-K_{\Sigma}-\sum_{k=1}^{i-1} \Delta_{k}\right), \mathcal{O}_{\Sigma}\right) .
$$

That is, there exists an extension

$$
0 \rightarrow \mathcal{O}_{\Sigma} \rightarrow E_{i} \rightarrow \mathcal{J}_{X_{i}^{0} / \Sigma}\left(D-K_{\Sigma}-\sum_{k=1}^{i-1} \Delta_{k}\right) \rightarrow 0
$$

The minimality of $X_{i}^{0}$ implies that $E_{i}$ is locally free and hence that $X_{i}^{0}$ is a locally complete intersection (cf. [Laz97]),

$$
c_{1}\left(E_{i}\right)=D-K_{\Sigma}-\sum_{k=1}^{i-1} \Delta_{k} \quad \text { and } \quad c_{2}\left(E_{i}\right)=\operatorname{deg}\left(X_{i}^{0}\right) .
$$

Step 4: $E_{i}$ is Bogomolov unstable.

According to the Bogomolov Theorem we only have to show that $c_{1}\left(E_{i}\right)^{2}>4 c_{2}\left(E_{i}\right)$ (cf. [Bog79] or [Laz97], Theorem 4.2). Since $(4 \beta-1) \cdot\left(D-K_{\Sigma}\right)^{2} \leq 0$ by (3) and since $\Delta_{k}^{2} \geq 0$ by Assumption $(*)$, we deduce:

$$
\begin{aligned}
4 c_{2}\left(E_{i}\right) & =4 \operatorname{deg}\left(X_{i}^{0}\right) \leq_{\text {Step } 2} 4 \operatorname{deg}\left(X_{0}\right)-4 \sum_{k=1}^{i-1} \operatorname{deg}\left(X_{k-1} \cap \Delta_{k}\right) \\
& <_{(3) /(\mathrm{c})} 4 \beta\left(D-K_{\Sigma}\right)^{2}-2 \sum_{k=1}^{i-1} \Delta_{k} \cdot\left(D-K_{\Sigma}-\sum_{j=1}^{k} \Delta_{j}\right)-2 \sum_{k=1}^{i-1} \Delta_{k}^{2} \\
& =\left(D-K_{\Sigma}-\sum_{k=1}^{i-1} \Delta_{k}\right)^{2}+(4 \beta-1) \cdot\left(D-K_{\Sigma}\right)^{2}-\sum_{k=1}^{i-1} \Delta_{k}^{2} \\
& \leq\left(D-K_{\Sigma}-\sum_{k=1}^{i-1} \Delta_{k}\right)^{2}=c_{1}\left(E_{i}\right)^{2} .
\end{aligned}
$$

Step 5: Find $\Delta_{i}$.

Since $E_{i}$ is Bogomolov unstable, there is a 0 -dimensional scheme $Z_{i} \subset \Sigma$ and a $\Delta_{i}^{0} \in \operatorname{Div}(\Sigma)$ such that

$$
0 \rightarrow \mathcal{O}_{\Sigma}\left(\Delta_{i}^{0}\right) \rightarrow E_{i} \rightarrow \mathcal{J}_{Z_{i} / \Sigma}\left(D-K_{\Sigma}-\sum_{k=1}^{i-1} \Delta_{k}-\Delta_{i}^{0}\right) \rightarrow 0
$$

is exact and such that

(d') $\left(2 \Delta_{i}^{0}-D+K_{\Sigma}+\sum_{k=1}^{i-1} \Delta_{k}\right)^{2} \geq c_{1}\left(E_{i}\right)^{2}-4 \cdot c_{2}\left(E_{i}\right)>0$, and

(e') $\left(2 \Delta_{i}^{0}-D+K_{\Sigma}+\sum_{k=1}^{i-1} \Delta_{k}\right) \cdot H>0$ for all $H \in \operatorname{Div}(\Sigma)$ ample.

Tensoring (4.4) with $\mathcal{O}_{\Sigma}\left(-\Delta_{i}^{0}\right)$ leads to the following exact sequence:

$$
0 \rightarrow \mathcal{O}_{\Sigma} \rightarrow E_{i}\left(-\Delta_{i}^{0}\right) \rightarrow \mathcal{J}_{Z_{i} / \Sigma}\left(D-K_{\Sigma}-\sum_{k=1}^{i-1} \Delta_{k}-2 \Delta_{i}^{0}\right) \rightarrow 0
$$

and we deduce that $h^{0}\left(\Sigma, E_{i}\left(-\Delta_{i}^{0}\right)\right) \neq 0$.

Now tensoring (4.2) with $\mathcal{O}_{\Sigma}\left(-\Delta_{i}^{0}\right)$ leads to

$$
0 \rightarrow \mathcal{O}_{\Sigma}\left(-\Delta_{i}^{0}\right) \rightarrow E_{i}\left(-\Delta_{i}^{0}\right) \rightarrow \mathcal{J}_{X_{i}^{0} / \Sigma}\left(D-K_{\Sigma}-\sum_{k=1}^{i-1} \Delta_{k}-\Delta_{i}^{0}\right) \rightarrow 0
$$


By $\left(\mathrm{e}^{\prime}\right)$, and (0), respectively (f),

$$
-\Delta_{i}^{0} \cdot H<-\frac{1}{2}\left(D-K_{\Sigma}-\sum_{k=1}^{i-1} \Delta_{k}\right) \cdot H \leq 0
$$

for an ample divisor $H$. Hence $-\Delta_{i}^{0}$ cannot be effective, that is, $H^{0}\left(\Sigma,-\Delta_{i}^{0}\right)=0$. But the long exact cohomology sequence of (4.6) then implies

$$
0 \neq H^{0}\left(\Sigma, E_{i}\left(-\Delta_{i}^{0}\right)\right) \hookrightarrow H^{0}\left(\Sigma, \mathcal{J}_{X_{i}^{0} / \Sigma}\left(D-K_{\Sigma}-\sum_{k=1}^{i-1} \Delta_{k}-\Delta_{i}^{0}\right)\right) .
$$

In particular, we may choose $\Delta_{i} \in\left|\mathcal{J}_{X_{i}^{0} / \Sigma}\left(D-K_{\Sigma}-\sum_{k=1}^{i-1} \Delta_{k}-\Delta_{i}^{0}\right)\right|_{l}$.

Step 6: $\Delta_{i}$ satisfies (d)-(f).

We note that by the choice of $\Delta_{i}$ we have the following equivalences:

$$
\begin{gathered}
\Delta_{i}^{0} \sim_{l} D-K_{\Sigma}-\sum_{k=1}^{i} \Delta_{k} \\
\Delta_{i}^{0}-\Delta_{i} \sim_{l} 2 \Delta_{i}^{0}-D+K_{\Sigma}+\sum_{k=1}^{i-1} \Delta_{k} \sim_{l} D-K_{\Sigma}-\sum_{k=1}^{i} \Delta_{k}-\Delta_{i} .
\end{gathered}
$$

Thus (d) and (e) is a reformulation of $\left(\mathrm{d}^{\prime}\right)$ and $\left(\mathrm{e}^{\prime}\right)$.

Moreover, since $\left(\Delta_{i}^{0}-\Delta_{i}\right) \cdot H>0$ for any ample $H$, we have $\left(\Delta_{i}^{0}-\Delta_{i}\right) \cdot H \geq 0$ for any $H$ in the closure of the ample cone; in particular,

$$
\Delta_{i}^{0} \cdot H \geq \Delta_{i} . H \geq 0 \quad \text { for all } H \text { nef, }
$$

since $\Delta_{i}$ is effective. Finally, since by assumption $(*)$ any effective divisor is nef, we deduce that $\Delta_{i}^{0}$. $C \geq 0$ for any curve $C$, that is, $\Delta_{i}^{0}$ is nef. In view of (4.7) for (f) it remains to show that $\left(\Delta_{i}^{0}\right)^{2}>0$. Once more taking into account that $\Delta_{i}$ is nef by $(*)$ we have by $\left(\mathrm{d}^{\prime}\right),(4.8)$, and (4.9),

$$
\left(\Delta_{i}^{0}\right)^{2}=\left(\Delta_{i}^{0}-\Delta_{i}\right)^{2}+\left(\Delta_{i}^{0}-\Delta_{i}\right) \cdot \Delta_{i}+\Delta_{i}^{0} \cdot \Delta_{i}>0 .
$$

Step 7: $\Delta_{i}$ satisfies (c).

We would like to apply the Bézout Theorem to $C_{0}$ and $\Delta_{i}$. Thus suppose that the irreducible curve $C_{0}$ is a component of $\Delta_{i}$ and let $H$ be any ample divisor.

Applying (d) and the fact that $D+K_{\Sigma}$ is nef by (0), we derive the contradiction

$$
0 \leq\left(\Delta_{i}-C_{0}\right) \cdot H<-\frac{1}{2} \cdot\left(D+K_{\Sigma}+\sum_{k=1}^{i-1} \Delta_{k}\right) \cdot H \leq-\frac{1}{2} \cdot\left(D+K_{\Sigma}\right) \cdot H \leq 0 .
$$

Since $X_{i-1} \subseteq X_{0} \subset C_{0}$, the Bézout Theorem therefore implies

$$
D . \Delta_{i}=C_{0} \cdot \Delta_{i} \geq \operatorname{deg}\left(X_{i-1} \cap \Delta_{i}\right) .
$$

By definition $X_{i}^{0} \subseteq X_{i-1}$ and $X_{i}^{0} \subset \Delta_{i}$. Thus

$$
\operatorname{deg}\left(X_{i-1} \cap \Delta_{i}\right) \geq \operatorname{deg}\left(X_{i}^{0}\right) .
$$

By assumption $(*)$ the curve $\Delta_{i}$ is nef and thus (4.9) gives

$$
\left(D-K_{\Sigma}-\sum_{k=1}^{i} \Delta_{k}\right) \cdot \Delta_{i}=\Delta_{i}^{0} \cdot \Delta_{i} \geq \Delta_{i}^{2} \geq 0
$$


Finally from $\left(\mathrm{d}^{\prime}\right)$ and by (4.3) it follows that

$$
\left(\Delta_{i}^{0}-\Delta_{i}\right)^{2} \geq c_{1}\left(E_{i}\right)^{2}-4 \cdot c_{2}\left(E_{i}\right)=\left(\Delta_{i}^{0}+\Delta_{i}\right)^{2}-4 \cdot \operatorname{deg}\left(X_{i}^{0}\right),
$$

and thus $\operatorname{deg}\left(X_{i}^{0}\right) \geq \Delta_{i}^{0} . \Delta_{i}$.

Step 8: After a finite number $m$ of steps, $h^{1}\left(\Sigma, \mathcal{J}_{X_{m} / \Sigma}\left(D-\sum_{i=1}^{m} \Delta_{i}\right)\right)=0$.

As we have mentioned in Step $1, \operatorname{deg}\left(X_{i}^{0}\right)>0$. This ensures that

$$
\operatorname{deg}\left(X_{i}\right)=\operatorname{deg}\left(X_{i-1}\right)-\operatorname{deg}\left(X_{i-1} \cap \Delta_{i}\right) \leq \operatorname{deg}\left(X_{i-1}\right)-\operatorname{deg}\left(X_{i}^{0}\right)<\operatorname{deg}\left(X_{i-1}\right),
$$

i.e., the degree of $X_{i}$ strictly decreases each time. Thus the procedure must stop after a finite number $m$ of steps

Step 9: It remains to show (4.1).

By assumption $(*)$ the curves $\Delta_{i}$ are nef; in particular, $\Delta_{i} . \Delta_{j} \geq 0$ for all $i, j$. Thus

(c) implies

$$
\begin{aligned}
\sum_{i=1}^{m} \operatorname{deg}\left(X_{i}^{0}\right) & \geq \sum_{i=1}^{m}\left(D-K_{\Sigma}-\sum_{k=1}^{i} \Delta_{k}\right) \cdot \Delta_{i} \\
& =\left(D-K_{\Sigma}\right) \cdot \sum_{i=1}^{m} \Delta_{i}-\frac{1}{2}\left(\left(\sum_{i=1}^{m} \Delta_{i}\right)^{2}+\sum_{i=1}^{m} \Delta_{i}^{2}\right) \\
& \geq\left(D-K_{\Sigma}\right) \cdot \sum_{i=1}^{m} \Delta_{i}-\left(\sum_{i=1}^{m} \Delta_{i}\right)^{2}
\end{aligned}
$$

But then, taking condition (3) into account,

$$
\begin{aligned}
0 & \leq \frac{1}{4}\left(D-K_{\Sigma}\right)^{2}-\operatorname{deg}\left(X_{0}\right) \leq \frac{1}{4}\left(D-K_{\Sigma}\right)^{2}-\sum_{i=1}^{m} \operatorname{deg}\left(X_{i}^{0}\right) \\
& \leq \frac{1}{4}\left(D-K_{\Sigma}\right)^{2}-\left(D-K_{\Sigma}\right) \cdot \sum_{i=1}^{m} \Delta_{i}+\left(\sum_{i=1}^{m} \Delta_{i}\right)^{2} \\
& =\left(\frac{1}{2}\left(D-K_{\Sigma}\right)-\sum_{i=1}^{m} \Delta_{i}\right)^{2} .
\end{aligned}
$$

It is our overall aim to compare the dimension of a cohomology group of the form $H^{1}\left(\Sigma, \mathcal{J}_{X_{0} / \Sigma}(D)\right)$ with some invariants of the $X_{i}^{0}$ and $\Delta_{i}$. The following lemma will be vital for the necessary estimates.

Lemma 4.2. Let $D \in \operatorname{Div}(\Sigma)$ and let $X_{0} \subset \Sigma$ be a zero-dimensional scheme such that there exist curves $\Delta_{1}, \ldots, \Delta_{m} \subset \Sigma$ and zero-dimensional schemes $X_{i}^{0} \subseteq X_{i-1}$ for $i=1, \ldots, m$, where $X_{i}=X_{i-1}: \Delta_{i}$ for $i=1, \ldots, m$, such that $(a)-(f)$ in Lemma 4.1 are fulfilled. 
Then:

$$
\begin{aligned}
h^{1}\left(\Sigma, \mathcal{J}_{X_{0} / \Sigma}(D)\right) & \leq \sum_{i=1}^{m} h^{1}\left(\Delta_{i}, \mathcal{J}_{X_{i-1} \cap \Delta_{i} / \Delta_{i}}\left(D-\sum_{k=1}^{i-1} \Delta_{k}\right)\right) \\
& \leq \sum_{i=1}^{m}\left(1+\operatorname{deg}\left(X_{i-1} \cap \Delta_{i}\right)-\operatorname{deg}\left(X_{i}^{0}\right)\right) \\
& \leq \sum_{i=1}^{m}\left(\Delta_{i} \cdot\left(K_{\Sigma}+\sum_{k=1}^{i} \Delta_{k}\right)+1\right) .
\end{aligned}
$$

Proof. Throughout the proof we use the following notation:

$$
\mathcal{G}_{i}=\mathcal{J}_{X_{i-1} \cap \Delta_{i} / \Delta_{i}}\left(D-\sum_{k=1}^{i-1} \Delta_{k}\right) \quad \text { and } \quad \mathcal{G}_{i}^{0}=\mathcal{J}_{X_{i}^{0} / \Delta_{i}}\left(D-\sum_{k=1}^{i-1} \Delta_{k}\right)
$$

for $i=1, \ldots, m$, and for $i=0, \ldots, m$,

$$
\mathcal{F}_{i}=\mathcal{J}_{X_{i} / \Sigma}\left(D-\sum_{k=1}^{i} \Delta_{k}\right) .
$$

Since $X_{i+1}=X_{i}: \Delta_{i+1}$, we have the following short exact sequence:

$$
0 \longrightarrow \mathcal{F}_{i+1} \stackrel{\cdot \Delta_{i+1}}{\longrightarrow} \mathcal{F}_{i} \longrightarrow \mathcal{G}_{i+1} \longrightarrow 0
$$

for $i=0, \ldots, m-1$ and the corresponding long exact cohomology sequence

$$
\begin{array}{r}
0 \longrightarrow H^{0}\left(\Sigma, \mathcal{F}_{i+1}\right) \longrightarrow H^{0}\left(\Sigma, \mathcal{F}_{i}\right) \rightarrow H^{0}\left(\Sigma, \mathcal{G}_{i+1}\right) \rightarrow H^{1}\left(\Sigma, \mathcal{F}_{i+1}\right) \\
\downarrow \\
0=H^{2}\left(\Sigma, \mathcal{G}_{i+1}\right) \leftarrow H^{2}\left(\Sigma, \mathcal{F}_{i}\right) \leftarrow H^{2}\left(\Sigma, \mathcal{F}_{i+1}\right) \leftarrow H^{1}\left(\Sigma, \mathcal{G}_{i+1}\right) \leftarrow H^{1}\left(\Sigma, \mathcal{F}_{i}\right)
\end{array}
$$

Step 1: $h^{1}\left(\Sigma, \mathcal{F}_{i}\right) \leq \sum_{j=i+1}^{m} h^{1}\left(\Sigma, \mathcal{G}_{j}\right)$ for $i=0, \ldots, m-1$.

We prove the claim by descending induction on $i$. From (4.11) we deduce

$$
0=H^{1}\left(\Sigma, \mathcal{F}_{m}\right) \rightarrow H^{1}\left(\Sigma, \mathcal{F}_{m-1}\right) \rightarrow H^{1}\left(\Sigma, \mathcal{G}_{m}\right),
$$

which implies $h^{1}\left(\Sigma, \mathcal{F}_{m-1}\right) \leq h^{1}\left(\Sigma, \mathcal{G}_{m}\right)$ and thus proves the case $i=m-1$.

We may therefore assume that $1 \leq i \leq m-2$. Once more from (4.11) we deduce

$$
a=h^{0}\left(\Sigma, \mathcal{F}_{i+1}\right)-h^{0}\left(\Sigma, \mathcal{F}_{i}\right)+h^{0}\left(\Sigma, \mathcal{G}_{i+1}\right) \geq 0
$$

and

and finally,

$$
b=h^{2}\left(\Sigma, \mathcal{F}_{i+1}\right)-h^{2}\left(\Sigma, \mathcal{F}_{i}\right) \geq 0
$$

$$
\begin{aligned}
h^{1}\left(\Sigma, \mathcal{F}_{i}\right) & =h^{1}\left(\Sigma, \mathcal{G}_{i+1}\right)+h^{1}\left(\Sigma, \mathcal{F}_{i+1}\right)-a-b \leq h^{1}\left(\Sigma, \mathcal{G}_{i+1}\right)+h^{1}\left(\Sigma, \mathcal{F}_{i+1}\right) \\
& \leq_{\text {Ind. }} h^{1}\left(\Sigma, \mathcal{G}_{i+1}\right)+\sum_{j=i+2}^{m} h^{1}\left(\Sigma, \mathcal{G}_{j}\right)=\sum_{j=i+1}^{m} h^{1}\left(\Sigma, \mathcal{G}_{j}\right) .
\end{aligned}
$$

Step 2: $h^{1}\left(\Delta_{i}, \mathcal{G}_{i}\right)=h^{0}\left(\Delta_{i}, \mathcal{G}_{i}\right)-\chi\left(\mathcal{O}_{\Delta_{i}}\left(D-\sum_{k=1}^{i-1} \Delta_{k}\right)\right)+\operatorname{deg}\left(X_{i-1} \cap \Delta_{i}\right)$.

We consider the exact sequence

$$
0 \rightarrow \mathcal{G}_{i} \longrightarrow \mathcal{O}_{\Delta_{i}}\left(D-\sum_{k=1}^{i-1} \Delta_{k}\right) \rightarrow \mathcal{O}_{X_{i-1} \cap \Delta_{i} / \Delta_{i}}\left(D-\sum_{k=1}^{i-1} \Delta_{k}\right) \rightarrow 0
$$

The result then follows from the long exact cohomology sequence. 
Step 3: $h^{0}\left(\Delta_{i}, \mathcal{G}_{i}^{0}\right)-\chi\left(\mathcal{O}_{\Delta_{i}}\left(D-\sum_{k=1}^{i-1} \Delta_{k}\right)\right)=h^{1}\left(\Delta_{i}, \mathcal{G}_{i}^{0}\right)-\operatorname{deg}\left(X_{i}^{0}\right)$.

This follows analogously, replacing $X_{i-1}$ by $X_{i}^{0}$, since $X_{i}^{0}=X_{i}^{0} \cap \Delta_{i}$.

Step 4: $h^{1}\left(\Delta_{i}, \mathcal{G}_{i}^{0}\right) \leq h^{1}\left(\Sigma, \mathcal{J}_{X_{i}^{0} / \Sigma}\left(D-\sum_{k=1}^{i-1} \Delta_{k}\right)\right)=1$.

Note that $X_{i}^{0}: \Delta_{i}=\emptyset$, and hence $\mathcal{J}_{X_{i}^{0}: \Delta_{i} / \Sigma}=\mathcal{O}_{\Sigma}$. We thus have the following short exact sequence:

$$
0 \longrightarrow \mathcal{O}_{\Sigma}\left(D-\sum_{k=1}^{i} \Delta_{k}\right) \stackrel{\Delta_{i}}{\longrightarrow} \mathcal{J}_{X_{i}^{0} / \Sigma}\left(D-\sum_{k=1}^{i-1} \Delta_{k}\right) \longrightarrow \mathcal{G}_{i}^{0} \longrightarrow 0 .
$$

By assumption (f) the divisor $D-K_{\Sigma}-\sum_{k=1}^{i} \Delta_{k}$ is big and nef and hence

$$
0=h^{0}\left(\Sigma, \mathcal{O}_{\Sigma}\left(-D+K_{\Sigma}+\sum_{k=1}^{i} \Delta_{k}\right)\right)=h^{2}\left(\Sigma, \mathcal{O}_{\Sigma}\left(D-\sum_{k=1}^{i} \Delta_{k}\right)\right) .
$$

Thus the long exact cohomology sequence of (4.12) gives

$$
H^{1}\left(\Sigma, \mathcal{J}_{X_{i}^{0} / \Sigma}\left(D-\sum_{k=1}^{i-1} \Delta_{k}\right)\right) \longrightarrow H^{1}\left(\Delta_{i}, \mathcal{G}_{i}^{0}\right) \longrightarrow 0
$$

and

$$
h^{1}\left(\Delta_{i}, \mathcal{G}_{i}^{0}\right) \leq h^{1}\left(\Sigma, \mathcal{J}_{X_{i}^{0} / \Sigma}\left(D-\sum_{k=1}^{i-1} \Delta_{k}\right)\right) .
$$

However, by assumption (b) the latter is just one.

Step 5: $h^{1}\left(\Delta_{i}, \mathcal{G}_{i}\right) \leq 1+\operatorname{deg}\left(X_{i-1} \cap \Delta_{i}\right)-\operatorname{deg}\left(X_{i}^{0}\right)$.

We note that $\mathcal{G}_{i} \hookrightarrow \mathcal{G}_{i}^{0}$, and thus $h^{0}\left(\Delta_{i}, \mathcal{G}_{i}\right) \leq h^{0}\left(\Delta_{i}, \mathcal{G}_{i}^{0}\right)$. But then

$$
\begin{array}{rll}
h^{1}\left(\Delta_{i}, \mathcal{G}_{i}\right) & \leq_{\text {Step 2/3 }} & h^{1}\left(\Delta_{i}, \mathcal{G}_{i}^{0}\right)-\operatorname{deg}\left(X_{i}^{0}\right)+\operatorname{deg}\left(X_{i-1} \cap \Delta_{i}\right) \\
& \leq_{\text {Step 4 }} & 1-\operatorname{deg}\left(X_{i}^{0}\right)+\operatorname{deg}\left(X_{i-1} \cap \Delta_{i}\right) .
\end{array}
$$

Step 6: Finish the proof.

Taking into account that $h^{1}\left(\Sigma, \mathcal{G}_{i}\right)=h^{1}\left(\Delta_{i}, \mathcal{G}_{i}\right)$, since $\mathcal{G}_{i}$ is concentrated on $\Delta_{i}$, the first inequality follows from Step 1, while the second inequality is a consequence of Step 5, and the last inequality follows from assumption (c).

In Lemmata 4.34 .4 and 4.6 we consider special classes of surfaces which allow us to do the necessary estimates in order to finally derive

$$
\sum_{i=1}^{m}\left(\# X_{i}^{0}-\operatorname{dim}\left|\Delta_{i}\right|_{l}\right)>h^{1}\left(\Sigma, \mathcal{J}_{X_{0} / \Sigma}(D)\right) .
$$

We first consider surfaces with Picard number one.

Lemma 4.3. Let $\Sigma$ be a surface such that

(i) $\mathrm{NS}(\Sigma)=L \cdot \mathbb{Z}$ and $L$ is ample, and

(ii) $h^{1}(\Sigma, C)=0$, whenever $C$ is effective.

Let $D \in \operatorname{Div}(\Sigma)$ and let $X_{0} \subset \Sigma$ be a zero-dimensional scheme satisfying (0)-(3) from Lemma 4.1 and

(4) $\sum_{z \in \Sigma}\left(\operatorname{deg}\left(X_{0, z}\right)\right)^{2}<\gamma \cdot\left(D-K_{\Sigma}\right)^{2}$, where $\gamma=\frac{(1+\sqrt{1-4 \beta})^{2} \cdot L^{2}}{4 \cdot \chi\left(\mathcal{O}_{\Sigma}\right)+\max \left\{0,2 \cdot K_{\Sigma} \cdot L\right\}+6 \cdot L^{2}}$. 
Then, using the notation of Lemma 4.1 and setting $X_{S}=\bigcup_{i=1}^{m} X_{i}^{0}$,

$$
h^{1}\left(\Sigma, \mathcal{J}_{X_{0} / \Sigma}(D)\right)+\sum_{i=1}^{m}\left(h^{0}\left(\Sigma, \mathcal{O}_{\Sigma}\left(\Delta_{i}\right)\right)-1\right)<\# X_{S}
$$

Proof. We fix the following notation:

$$
D \sim_{a} d \cdot L, \quad K_{\Sigma} \sim_{a} \kappa \cdot L, \quad \Delta_{i} \sim_{a} \delta_{i} \cdot L, \quad \text { and } l=\sqrt{L^{2}}>0 .
$$

Furthermore, we have $\gamma=\frac{(1+\sqrt{1-4 \beta})^{2}}{4 \alpha}$, where

$$
\alpha=\frac{4 \cdot \chi\left(\mathcal{O}_{\Sigma}\right)+\max \left\{0,2 \cdot K_{\Sigma} \cdot L\right\}+6 \cdot L^{2}}{4 \cdot L^{2}}= \begin{cases}\frac{\chi\left(\mathcal{O}_{\Sigma}\right)}{l^{2}}+\frac{\kappa+3}{2}, & \text { if } \kappa \geq 0 \\ \frac{\chi\left(\mathcal{O}_{\Sigma}\right)}{l^{2}}+\frac{3}{2}, & \text { if } \kappa<0 .\end{cases}
$$

Step 1: By (i) $\Sigma$ satisfies assumption $(*)$ of Lemma 4.1.

Step 2: $\sum_{i=1}^{m} \delta_{i} \cdot l \leq \frac{(d-\kappa) \cdot l}{2}-\sqrt{\frac{(d-\kappa)^{2} \cdot l^{2}}{4}-\operatorname{deg}\left(X_{S}\right)}$, by (4.1).

Step 3: $h^{1}\left(\Sigma, \mathcal{J}_{X_{0}}(D)\right) \leq\left(\kappa \cdot \sum_{i=1}^{m} \delta_{i}\right) \cdot l^{2}+\frac{1}{2}\left(\left(\sum_{i=1}^{m} \delta_{i}\right)^{2}+\sum_{i=1}^{m} \delta_{i}^{2}\right) \cdot l^{2}+m$.

By Lemma 4.2 we know that

$$
\begin{aligned}
h^{1}\left(\Sigma, \mathcal{J}_{X_{0}}(D)\right) & \leq \sum_{i=1}^{m}\left(\Delta_{i} \cdot\left(K_{\Sigma}+\sum_{k=1}^{i} \Delta_{k}\right)+1\right) \\
& =\left(\kappa \cdot \sum_{i=1}^{m} \delta_{i}\right) \cdot l^{2}+\frac{1}{2}\left(\left(\sum_{i=1}^{m} \delta_{i}\right)^{2}+\sum_{i=1}^{m} \delta_{i}^{2}\right) \cdot l^{2}+m .
\end{aligned}
$$

Step 4: $\sum_{i=1}^{m}\left(h^{0}\left(\Sigma, \mathcal{O}_{\Sigma}\left(\Delta_{i}\right)\right)-1\right) \leq m \cdot\left(\chi\left(\mathcal{O}_{\Sigma}\right)-1\right)+\frac{l^{2}}{2} \cdot \sum_{i=1}^{m} \delta_{i}^{2}-\frac{\kappa \cdot l^{2}}{2} \cdot \sum_{i=1}^{m} \delta_{i}$.

Since $\Delta_{i}$ is effective by (ii), $h^{1}\left(\Sigma, \Delta_{i}\right)=0$. Hence by Riemann-Roch

$$
\begin{aligned}
\sum_{i=1}^{m}\left(h^{0}\left(\Sigma, \mathcal{O}_{\Sigma}\left(\Delta_{i}\right)\right)-1\right) & \leq-m+m \cdot \chi\left(\mathcal{O}_{\Sigma}\right)+\frac{1}{2} \sum_{i=1}^{m}\left(\Delta_{i}^{2}-K_{\Sigma} \cdot \Delta_{i}\right) \\
& =m \cdot\left(\chi\left(\mathcal{O}_{\Sigma}\right)-1\right)+\frac{l^{2}}{2} \cdot \sum_{i=1}^{m} \delta_{i}^{2}-\frac{\kappa \cdot l^{2}}{2} \cdot \sum_{i=1}^{m} \delta_{i}
\end{aligned}
$$

Step 5: Finish the proof. 
In the following consideration we use that $\operatorname{deg}\left(X_{S}\right) \leq \operatorname{deg}\left(X_{0}\right) \leq \beta \cdot(d-\kappa)^{2} \cdot l^{2}$ :

$$
\begin{aligned}
& h^{1}\left(\Sigma, \mathcal{J}_{X_{0}}(D)\right)+\sum_{i=1}^{m}\left(h^{0}\left(\Sigma, \mathcal{O}_{\Sigma}\left(\Delta_{i}\right)\right)-1\right) \\
& \leq_{\text {Step 3 / }} m \cdot \chi\left(\mathcal{O}_{\Sigma}\right)+l^{2} \cdot \sum_{i=1}^{m} \delta_{i}^{2}+\frac{\kappa \cdot l^{2}}{2} \cdot \sum_{i=1}^{m} \delta_{i}+\frac{l^{2}}{2} \cdot\left(\sum_{i=1}^{m} \delta_{i}\right)^{2} \\
& \leq \alpha \cdot\left(l \cdot \sum_{i=1}^{m} \delta_{i}\right)^{2} \leq_{\text {Step } 2} \alpha \cdot\left(\frac{(d-\kappa) \cdot l}{2}-\sqrt{\frac{(d-\kappa)^{2} \cdot l^{2}}{4}-\operatorname{deg}\left(X_{S}\right)}\right)^{2} \\
& \leq \alpha \cdot\left(\frac{\frac{(d-\kappa)^{2} \cdot l^{2}}{4}-\left(\frac{(d-\kappa)^{2} \cdot l^{2}}{4}-\operatorname{deg}\left(X_{S}\right)\right)}{\frac{(d-\kappa) \cdot l}{2}+\sqrt{\frac{(d-\kappa)^{2} \cdot l^{2}}{4}-\operatorname{deg}\left(X_{S}\right)}}\right)^{2} \\
& =\alpha \cdot\left(\frac{\left.2 \cdot \operatorname{deg}\left(X_{S}\right)\right)}{(d-\kappa) \cdot l+\sqrt{(d-\kappa)^{2} \cdot l^{2}-4 \cdot \operatorname{deg}\left(X_{S}\right)}}\right)^{2} \\
& \leq \frac{4 \alpha}{(1+\sqrt{1-4 \beta})^{2} \cdot(d-\kappa)^{2} \cdot l^{2}} \cdot\left(\operatorname{deg}\left(X_{S}\right)\right)^{2} \\
& =\frac{1}{\gamma \cdot\left(D-K_{\Sigma}\right)^{2}} \cdot\left(\sum_{z \in \Sigma} \operatorname{deg}\left(X_{S, z}\right)\right)^{2} \\
& \leq \frac{\# X_{S}}{\gamma \cdot\left(D-K_{\Sigma}\right)^{2}} \cdot \sum_{z \in \Sigma} \operatorname{deg}\left(X_{S, z}\right)^{2} \\
& \leq \frac{\# X_{S}}{\gamma \cdot\left(D-K_{\Sigma}\right)^{2}} \cdot \sum_{z \in \Sigma} \operatorname{deg}\left(X_{0, z}\right)^{2}<_{(4)} \# X_{S} \text {. }
\end{aligned}
$$

The second class of surfaces that we consider are products of curves.

Lemma 4.4. Let $C_{1}$ and $C_{2}$ be two smooth projective curves of genera $g_{1}$ and $g_{2}$, respectively, with $g_{1} \geq g_{2} \geq 0$, such that for $\Sigma=C_{1} \times C_{2}$, the Néron-Severi group is $\operatorname{NS}(\Sigma)=C_{1} \mathbb{Z} \oplus C_{2} \mathbb{Z}$, and let $D \in \operatorname{Div}(\Sigma)$ be such that $D \sim_{a} a C_{1}+b C_{2}$ with $a>\max \left\{2 g_{2}-2,2-2 g_{2}\right\}$ and $b>\max \left\{2 g_{1}-2,2-2 g_{1}\right\}$. Suppose moreover that $X_{0} \subset \Sigma$ is a zero-dimensional scheme satisfying (1)-(3) from Lemma 4.1 and

(4) $\sum_{z \in \Sigma}\left(\operatorname{deg}\left(X_{0, z}\right)\right)^{2}<\gamma \cdot\left(D-K_{\Sigma}\right)^{2}$,

where $\gamma$ may be taken from the table in Theorem 2.7.

Then, using the notation of Lemma 4.1 and setting $X_{S}=\bigcup_{i=1}^{m} X_{i}^{0}$,

$$
h^{1}\left(\Sigma, \mathcal{J}_{X_{0}}(D)\right)+\sum_{i=1}^{m}\left(h^{0}\left(\Sigma, \mathcal{O}_{\Sigma}\left(\Delta_{i}\right)\right)-1\right)<\# X_{S} .
$$

Proof. Then $K_{\Sigma} \sim_{a}\left(2 g_{2}-2\right) \cdot C_{1}+\left(2 g_{1}-2\right) \cdot C_{2}$ and we fix the notation:

$$
\Delta_{i} \sim_{a} a_{i} C_{1}+b_{i} C_{2}, \quad \kappa_{1}=a-2 g_{2}+2 \quad \text { and } \quad \kappa_{2}=b-2 g_{1}+2 .
$$

Step 1: $\Sigma$ satisfies assumption $(*)$ of Lemma 4.1, Moreover, due to the assumptions on $a$ and $b$ we know that $D-K_{\Sigma}$ is ample and $D+K_{\Sigma}$ is nef, i.e., (0) in Lemma 4.1 is fulfilled as well.

Step 2a: $\left(\frac{\kappa_{1}}{4}\right) \cdot \sum_{i=1}^{m} b_{i}+\left(\frac{\kappa_{2}}{4}\right) \cdot \sum_{i=1}^{m} a_{i} \leq \operatorname{deg}\left(X_{S}\right)$. 
Let us first notice that the strict inequality " $<$ " in Lemma4.1 (e) for ample divisors $H$ comes down to " $\leq$ " for nef divisors $H$. We may apply this for $H=C_{1}$ and $H=C_{2}$ and deduce the following inequalities:

$$
0 \leq\left(D-K_{\Sigma}-\sum_{k=1}^{i} \Delta_{k}-\Delta_{i}\right) \cdot C_{1}=\kappa_{2}-\sum_{k=1}^{i} b_{k}-b_{i}
$$

and

$$
0 \leq\left(D-K_{\Sigma}-\sum_{k=1}^{i} \Delta_{k}-\Delta_{i}\right) \cdot C_{2}=\kappa_{1}-\sum_{k=1}^{i} a_{k}-a_{i} .
$$

For the following consideration we choose $i_{0}, j_{0} \in\{1, \ldots, m\}$ such that $a_{i_{0}} \geq a_{i}$ for all $i=1, \ldots, m$ and $b_{j_{0}} \geq b_{j}$ for all $j=1, \ldots, m$. Then

$$
\kappa_{1} \geq 2 a_{i} \quad \text { and } \quad \kappa_{2} \geq 2 b_{j}
$$

for all $i, j=1, \ldots, m$; finally (4.13) 4.15 lead to

$$
\begin{aligned}
& \operatorname{deg}\left(X_{S}\right)=\sum_{i=1}^{m} \operatorname{deg}\left(X_{i}^{0}\right) \geq_{\text {Lemmat4.1 (c) }} \sum_{i=1}^{m}\left(D-K_{\Sigma}-\sum_{k=1}^{i} \Delta_{k}\right) \cdot \Delta_{i} \\
& =\kappa_{1} \sum_{i=1}^{m} b_{i}+\kappa_{2} \sum_{i=1}^{m} a_{i}-\sum_{i=1}^{m} a_{i} b_{i}-\sum_{i=1}^{m} a_{i} \sum_{i=1}^{m} b_{i} \\
& \geq \frac{\kappa_{1}}{2} \sum_{i=1}^{m} b_{i}+\frac{\kappa_{2}}{2} \sum_{i=1}^{m} a_{i}+\frac{a_{m}}{2} \sum_{i=1}^{m} b_{i}+\frac{b_{m}}{2} \sum_{i=1}^{m} a_{i}-\sum_{i=1}^{m} a_{i} b_{i} \\
& \geq \frac{\kappa_{1}}{4} \sum_{i=1}^{m} b_{i}+\frac{\kappa_{2}}{4} \sum_{i=1}^{m} a_{i} .
\end{aligned}
$$

Step 2b: $\sum_{i=1}^{m} a_{i} \cdot \sum_{i=1}^{m} b_{i} \leq \frac{8}{\left(D-K_{\Sigma}\right)^{2}} \cdot\left(\operatorname{deg}\left(X_{S}\right)\right)^{2}$.

Using Step 2a we deduce

$$
\begin{aligned}
\left(\operatorname{deg}\left(X_{S}\right)\right)^{2} & >\left(\frac{\kappa_{2}}{4} \cdot \sum_{i=1}^{m} a_{i}+\frac{\kappa_{1}}{4} \cdot \sum_{i=1}^{m} b_{i}\right)^{2} \\
& \geq \frac{4 \cdot \kappa_{1} \cdot \kappa_{2}}{16} \cdot \sum_{i=1}^{m} a_{i} \cdot \sum_{i=1}^{m} b_{i} \\
& =\frac{\left(D-K_{\Sigma}\right)^{2}}{8} \cdot \sum_{i=1}^{m} a_{i} \cdot \sum_{i=1}^{m} b_{i} .
\end{aligned}
$$

Step 2c: $\sum_{i=1}^{m} a_{i} \leq \begin{cases}\frac{2 \alpha}{\left(D-K_{\Sigma}\right)^{2}} \cdot\left(\operatorname{deg}\left(X_{S}\right)\right)^{2}, & \text { if } \sum_{i=1}^{m} b_{i}=0, \\ \frac{8}{\left(D-K_{\Sigma}\right)^{2}} \cdot\left(\operatorname{deg}\left(X_{S}\right)\right)^{2}, & \text { otherwise. }\end{cases}$ If $\sum_{i=1}^{m} b_{i}=0$, then the same consideration as in Step 2a shows that

$$
\operatorname{deg}\left(X_{S}\right) \geq \kappa_{2} \cdot \sum_{i=1}^{m} a_{i}>0
$$

and thus

$$
\frac{\left(D-K_{\Sigma}\right)^{2}}{2 \alpha} \cdot \sum_{i=1}^{m} a_{i} \leq \kappa_{2}^{2} \cdot\left(\sum_{i=1}^{m} a_{i}\right)^{2} \leq\left(\operatorname{deg}\left(X_{S}\right)\right)^{2} .
$$


If $\sum_{i=1}^{m} b_{i} \neq 0$, then we are done by Step $2 \mathrm{~b}$.

Step 2d: $\sum_{i=1}^{m} b_{i} \leq \begin{cases}\frac{2}{\alpha \cdot\left(D-K_{\Sigma}\right)^{2}} \cdot\left(\operatorname{deg}\left(X_{S}\right)\right)^{2}, & \text { if } \sum_{i=1}^{m} a_{i}=0, \\ \frac{8}{\left(D-K_{\Sigma}\right)^{2}} \cdot\left(\operatorname{deg}\left(X_{S}\right)\right)^{2}, & \text { otherwise. }\end{cases}$

This is proved in the same way as Step 2c.

Step 3: $h^{1}\left(\Sigma, \mathcal{J}_{X_{0}}(D)\right) \leq 2 \sum_{i=1}^{m} a_{i} \sum_{i=1}^{m} b_{i}+\left(2 g_{1}-2\right) \sum_{i=1}^{m} a_{i}+\left(2 g_{2}-2\right) \sum_{i=1}^{m} b_{i}+$ $m$.

The following sequence of inequalities is due to Lemma 4.2 and the fact that $\Delta_{i} . \Delta_{j} \geq 0$ for any $i, j \in\{1, \ldots, m\}$ :

$$
\begin{aligned}
& h^{1}\left(\Sigma, \mathcal{J}_{X_{0}}(D)\right) \leq \sum_{i=1}^{m}\left(\Delta_{i} \cdot\left(K_{\Sigma}+\sum_{k=1}^{i} \Delta_{k}\right)+1\right) \\
& \quad \leq K_{\Sigma} \cdot \sum_{i=1}^{m} \Delta_{i}+\left(\sum_{i=1}^{m} \Delta_{i}\right)^{2}+m \\
& \quad=\left(2 g_{1}-2\right) \cdot \sum_{i=1}^{m} a_{i}+\left(2 g_{2}-2\right) \cdot \sum_{i=1}^{m} b_{i}+2 \cdot \sum_{i=1}^{m} a_{i} \cdot \sum_{i=1}^{m} b_{i}+m .
\end{aligned}
$$

Step 4: We find the estimate $\sum_{i=1}^{m}\left(h^{0}\left(\Sigma, \mathcal{O}_{\Sigma}\left(\Delta_{i}\right)\right)-1\right) \leq \beta$, where

$$
\beta=\left\{\begin{array}{l}
\sum_{i=1}^{m} a_{i} \cdot \sum_{i=1}^{m} b_{i}+\sum_{i=1}^{m} b_{i}, \quad \text { if } g_{1}=1, g_{2}=0, \\
\sum_{i=1}^{m} a_{i} \cdot \sum_{i=1}^{m} b_{i}-m, \quad \text { if } g_{1}=1, g_{2}=1, \exists i_{0}: a_{i_{0}} b_{i_{0}}>0 \\
\sum_{i=1}^{m} a_{i}+\sum_{i=1}^{m} b_{i}-m, \quad \text { if } g_{1}=1, g_{2}=1, \forall i: a_{i} b_{i}=0 \\
\sum_{i=1}^{m} a_{i} \cdot \sum_{i=1}^{m} b_{i}+\sum_{i=1}^{m} a_{i}+\sum_{i=1}^{m} b_{i}, \quad \text { otherwise. }
\end{array}\right.
$$

In general $h^{0}\left(\Sigma, \mathcal{O}_{\Sigma}\left(\Delta_{i}\right)\right) \leq a_{i} b_{i}+a_{i}+b_{i}+1$, whereas if $g_{1}=1, g_{2}=0$, we have $h^{0}\left(\Sigma, \mathcal{O}_{\Sigma}\left(\Delta_{i}\right)\right)=a_{i} b_{i}+b_{i}+1$. It thus only remains to consider the case $g_{1}=g_{2}=1$, where we get

$$
\sum_{i=1}^{m} h^{0}\left(\Sigma, \mathcal{O}_{\Sigma}\left(\Delta_{i}\right)\right)=\sum_{a_{i}, b_{i}>0} a_{i} b_{i}+\sum_{a_{i}=0} b_{i}+\sum_{b_{i}=0} a_{i} .
$$

If always either $a_{i}$ or $b_{i}$ is zero, we are done. Otherwise there exists some $i_{0} \in$ $\{1, \ldots, m\}$ such that $a_{i_{0}} \neq 0 \neq b_{i_{0}}$. Then looking at the right-hand side we see

$$
\sum_{i=1}^{m} h^{0}\left(\Sigma, \mathcal{O}_{\Sigma}\left(\Delta_{i}\right)\right) \leq \sum_{a_{i}, b_{i}>0} a_{i} b_{i}+a_{i_{0}} \cdot \sum_{a_{i}=0} b_{i}+b_{i_{0}} \cdot \sum_{b_{i}=0} a_{i} \leq \sum_{i=1}^{m} a_{i} \cdot \sum_{i=1}^{m} b_{i} .
$$

Step 5: Finish the proof.

Using Step 3 and Step 4, and taking $m \leq \sum_{i=1}^{m} a_{i}+b_{i}$ into account, we get $h^{1}\left(\Sigma, \mathcal{J}_{X_{0}}(D)\right)+\sum_{i=1}^{m}\left(h^{0}\left(\Sigma, \mathcal{O}_{\Sigma}\left(\Delta_{i}\right)\right)-1\right) \leq \beta^{\prime}$, where $\beta^{\prime}$ may be chosen as

$$
\beta^{\prime}= \begin{cases}3 \cdot \sum_{i=1}^{m} a_{i} \cdot \sum_{i=1}^{m} b_{i}, & \text { if } g_{1}=0, g_{2}=0 \\ 3 \cdot \sum_{i=1}^{m} a_{i} \cdot \sum_{i=1}^{m} b_{i}+\sum_{i=1}^{m} a_{i}, & \text { if } g_{1}=1, g_{2}=0 \\ 3 \cdot \sum_{i=1}^{m} a_{i} \cdot \sum_{i=1}^{m} b_{i}+2 g_{1} \cdot \sum_{i=1}^{m} a_{i}+2 g_{2} \cdot \sum_{i=1}^{m} b_{i}, & \text { if } g_{1} \geq 2, g_{2} \geq 0\end{cases}
$$


For the case $g_{1}=g_{2}=1$ we take a closer look. We find at once the following upper bounds $\beta^{\prime \prime}$ for $h^{1}\left(\Sigma, \mathcal{J}_{X_{0}}(D)\right)+\sum_{i=1}^{m}\left(h^{0}\left(\Sigma, \mathcal{O}_{\Sigma}\left(\Delta_{i}\right)\right)-1\right)$ :

$$
\beta^{\prime \prime}= \begin{cases}3 \cdot \sum_{i=1}^{m} a_{i} \cdot \sum_{i=1}^{m} b_{i}, & \text { if } \exists i_{0}: a_{i_{0}} b_{i_{0}} \neq 0, \\ 2 \cdot \sum_{i=1}^{m} a_{i} \cdot \sum_{i=1}^{m} b_{i}+\sum_{i=1}^{m} a_{i}+\sum_{i=1}^{m} b_{i}, & \text { if } \forall i: a_{i} b_{i}=0 .\end{cases}
$$

Considering now the cases $\sum_{i=1}^{m} a_{i} \neq 0 \neq \sum_{i=1}^{m} b_{i}, \sum_{i=1}^{m} a_{i}=0$ and $\sum_{i=1}^{m} b_{i}=0$, we can replace these by

$$
\beta^{\prime \prime} \leq \beta^{\prime}= \begin{cases}4 \cdot \sum_{i=1}^{m} a_{i} \cdot \sum_{i=1}^{m} b_{i}, & \text { if } \sum_{i=1}^{m} a_{i} \neq 0 \neq \sum_{i=1}^{m} b_{i}, \\ \sum_{i=1}^{m} a_{i}, & \text { if } \sum_{i=1}^{m} b_{i}=0 \\ \sum_{i=1}^{m} b_{i}, & \text { if } \sum_{i=1}^{m} a_{i}=0\end{cases}
$$

Applying now the results of Step 2 in all cases we get

$$
\begin{aligned}
& h^{1}(\Sigma,\left.\mathcal{J}_{X_{0}}(D)\right)+\sum_{i=1}^{m}\left(h^{0}\left(\Sigma, \mathcal{O}_{\Sigma}\left(\Delta_{i}\right)\right)-1\right) \leq \beta^{\prime} \leq \frac{1}{\gamma \cdot\left(D-K_{\Sigma}\right)^{2}} \cdot\left(\operatorname{deg}\left(X_{S}\right)\right)^{2} \\
& \quad=\frac{1}{\gamma \cdot\left(D-K_{\Sigma}\right)^{2}} \cdot\left(\sum_{z \in \Sigma} \operatorname{deg}\left(X_{S, z}\right)\right)^{2} \leq \frac{\# X_{S}}{\gamma \cdot\left(D-K_{\Sigma}\right)^{2}} \cdot \sum_{z \in \Sigma} \operatorname{deg}\left(X_{S, z}\right)^{2} \\
& \quad \leq \frac{\# X_{S}}{\gamma \cdot\left(D-K_{\Sigma}\right)^{2}} \cdot \sum_{z \in \Sigma} \operatorname{deg}\left(X_{0, z}\right)^{2}<_{(4)} \# X_{S}
\end{aligned}
$$

Remark 4.5. Lemma 4.4 and hence Theorem 2.7 could easily be generalised to other surfaces $\Sigma$ with irreducible curves $C_{1}, C_{2} \subset \Sigma$ such that $\mathrm{NS}(\Sigma)=C_{1} \mathbb{Z} \oplus C_{2} \mathbb{Z}$ with intersection matrix $\left(\begin{array}{ll}0 & 1 \\ 1 & 0\end{array}\right)$ once we have an estimate similar to

$$
h^{0}\left(\Sigma, a C_{1}+b C_{2}\right) \leq a b+a+b+1
$$

for an effective divisor $a C_{1}+b C_{2}$.

With a number of small modifications, we are even able to adapt it in the following lemma in the case of geometrically ruled surfaces with non-positive invariant $e$ although the intersection pairing looks more complicated.

The problem with arbitrary geometrically ruled surfaces is the existence of the section with negative self-intersection, once the invariant $e>0$, since then the proof of Lemma 4.1 no longer works.

In the following lemma we use the notation of Subsection 2.3.

Lemma 4.6. Let $\pi: \Sigma \rightarrow C$ be a geometrically ruled surface with invariant $e \leq 0$ and $g=g(C)$, and let $D \in \operatorname{Div}(\Sigma)$ be such that $D \sim_{a} a C_{0}+b F$ with $a \geq 2$, $b>2 g-2+\frac{a e}{2}$, and if $g=0$, then $b \geq 2$. Suppose moreover that $X_{0} \subset \Sigma$ is a zero-dimensional scheme satisfying (1)-(3) from Lemma 4.1 and

(4) $\sum_{z \in \Sigma}\left(\operatorname{deg}\left(X_{0, z}\right)\right)^{2}<\gamma \cdot\left(D-K_{\Sigma}\right)^{2}$, where $\gamma$ may be taken from the table in Theorem 2.8 .

Then, using the notation of Lemma 4.1 and setting $X_{S}=\bigcup_{i=1}^{m} X_{i}^{0}$,

$$
h^{1}\left(\Sigma, \mathcal{J}_{X_{0}}(D)\right)+\sum_{i=1}^{m}\left(h^{0}\left(\Sigma, \mathcal{O}_{\Sigma}\left(\Delta_{i}\right)\right)-1\right)<\# X_{S} .
$$


Proof. Remember that the Néron-Severi group of $\Sigma$ is generated by a section $C_{0}$ of $\pi$ and a fibre $F$ with intersection pairing given by $\left(\begin{array}{rr}-e & 1 \\ 1 & 0\end{array}\right)$. Then $K_{\Sigma} \sim_{a}-2 C_{0}+$ $(2 g-2-e) \cdot F$ and we fix the notation:

$$
\Delta_{i} \sim_{a} a_{i} C_{0}+b_{i}^{\prime} F
$$

Note that then

$$
a_{i} \geq 0 \quad \text { and } \quad b_{i}:=b_{i}^{\prime}-\frac{e}{2} a_{i} \geq 0 .
$$

Finally, we set $\kappa_{1}=a+2$ and $\kappa_{2}=b+2-2 g-\frac{a e}{2}$ and get

$$
\left(D-K_{\Sigma}\right)^{2}=-e \cdot(a+2)^{2}+2 \cdot(a+2) \cdot(b+2+e-2 g)=2 \cdot \kappa_{1} \cdot \kappa_{2} .
$$

Replacing the equations (4.13) and (4.14) by

$$
0 \leq\left(D-K_{\Sigma}-\sum_{k=1}^{i} \Delta_{k}-\Delta_{i}\right) \cdot\left(C_{0}+\frac{e}{2} F\right)=\kappa_{2}-\sum_{k=1}^{i} b_{k}-b_{i}
$$

and

$$
0 \leq\left(D-K_{\Sigma}-\sum_{k=1}^{i} \Delta_{k}-\Delta_{i}\right) \cdot F=\kappa_{1}-\sum_{k=1}^{i} a_{k}-a_{i},
$$

the assertions of Step 1 to Step 2c in the proof of Lemma 4.4 remain literally true. Step 2d: $\left(\sum_{i=1}^{m} a_{i}\right)^{2} \leq \frac{32 \alpha}{\left(D-K_{\Sigma}\right)^{2}}\left(\operatorname{deg}\left(X_{S}\right)\right)^{2}$ and $\left(\sum_{i=1}^{m} b_{i}\right)^{2} \leq \frac{32}{\alpha \cdot\left(D-K_{\Sigma}\right)^{2}}\left(\operatorname{deg}\left(X_{S}\right)\right)^{2}$. This follows from the following inequality with the aid of Step 2a and 4.16):

$$
\begin{aligned}
\left(\operatorname{deg}\left(X_{S}\right)\right)^{2} \geq\left(\frac{\kappa_{2}}{4} \cdot \sum_{i=1}^{m} a_{i}\right)^{2}+ & \left(\frac{\kappa_{1}}{4} \cdot \sum_{i=1}^{m} b_{i}\right)^{2} \\
& \geq \frac{2 \cdot \kappa_{1} \cdot \kappa_{2}}{32 \alpha} \cdot\left(\sum_{i=1}^{m} a_{i}\right)^{2}+\frac{2 \cdot \kappa_{1} \cdot \kappa_{2} \cdot \alpha}{32} \cdot\left(\sum_{i=1}^{m} b_{i}\right)^{2} .
\end{aligned}
$$

Step 3: $h^{1}\left(\Sigma, \mathcal{J}_{X_{0}}(D)\right) \leq 2 \cdot \sum_{i=1}^{m} a_{i} \cdot \sum_{i=1}^{m} b_{i}+(2 g-2) \cdot \sum_{i=1}^{m} a_{i}-2 \cdot \sum_{i=1}^{m} b_{i}+m$ is proved as Step 3 in Lemma 4.4 .

Step 4a: If $e=0$, we find the estimate

$$
\sum_{i=1}^{m}\left(h^{0}\left(\Sigma, \mathcal{O}_{\Sigma}\left(\Delta_{i}\right)\right)-1\right) \leq \begin{cases}\sum_{i=1}^{m} a_{i} \cdot \sum_{i=1}^{m} b_{i}+\sum_{i=1}^{m} b_{i}-m, & \text { if } g=1, \sum_{i=1}^{m} b_{i} \neq 0 \\ \sum_{i=1}^{m} a_{i} \cdot \sum_{i=1}^{m} b_{i}+\sum_{i=1}^{m} b_{i}=0, & \text { if } g=1, \sum_{i=1}^{m} b_{i}=0 \\ \sum_{i=1}^{m} a_{i} \cdot \sum_{i=1}^{m} b_{i}+\sum_{i=1}^{m} a_{i}+\sum_{i=1}^{m} b_{i}, & \text { for } g \text { arbitrary. }\end{cases}
$$

We note that in this case $b_{i}^{\prime}=b_{i}$ and that $b_{i}=0$ thus implies $a_{i}>0$. But then

$$
h^{0}\left(\Sigma, \mathcal{O}_{\Sigma}\left(\Delta_{i}\right)\right) \leq \begin{cases}a_{i} b_{i}+b_{i}, & \text { if } g=1, b_{i}>0 \\ a_{i} b_{i}+b_{i}+1=1, & \text { if } g=1, b_{i}=0 \\ a_{i} b_{i}+a_{i}+b_{i}+1, & \text { otherwise }\end{cases}
$$

The results for $g$ arbitrary, respectively, $g=1$ and $\sum_{i=1}^{m} b_{i}=0$ thus follow right away. If, however, some $b_{i_{0}}>0$, then $\sum_{i \neq j} a_{i} b_{j} \geq b_{i_{0}} \sum_{i \neq i_{0}} a_{i} \geq \#\left\{b_{i} \mid b_{i}=0\right\}$ 
and hence

$$
\begin{aligned}
& h^{0}\left(\Sigma, \mathcal{O}_{\Sigma}\left(\Delta_{i}\right)\right) \leq \sum_{i=1}^{m} a_{i} b_{i}+\sum_{i=1}^{m} b_{i}+\#\left\{b_{i} \mid b_{i}=0\right\} \\
& \quad=\sum_{i=1}^{m} a_{i} \cdot \sum_{i=1}^{m} b_{i}+\sum_{i=1}^{m} b_{i}+\#\left\{b_{i} \mid b_{i}=0\right\}-\sum_{i \neq j} a_{i} b_{j} \leq \sum_{i=1}^{m} a_{i} \cdot \sum_{i=1}^{m} b_{i}+\sum_{i=1}^{m} b_{i} .
\end{aligned}
$$

Step 4b: If $e<0$, we give several upper bounds for $\beta=\sum_{i=1}^{m}\left(h^{0}\left(\Sigma, \mathcal{O}_{\Sigma}\left(\Delta_{i}\right)\right)-1\right)$ :

$$
\beta \leq\left\{\begin{array}{l}
\frac{1}{2} \sum_{i=1}^{m} a_{i} \sum_{i=1}^{m} b_{i}+\frac{1}{2}\left(\sum_{i=1}^{m} b_{i}\right)^{2}+\frac{1}{8}\left(\sum_{i=1}^{m} a_{i}\right)^{2}+\frac{1}{4} \sum_{i=1}^{m} a_{i}+\frac{1}{2} \sum_{i=1}^{m} b_{i}, \text { if } g=1, \\
\sum_{i=1}^{m} a_{i} \sum_{i=1}^{m} b_{i}+\sum_{i=1}^{m} a_{i}+\sum_{i=1}^{m} b_{i}-\frac{9 e}{32}\left(\sum_{i=1}^{m} a_{i}\right)^{2}, \quad \text { for } g \text { arbitrary, } \\
\frac{1}{4} \sum_{i=1}^{m} a_{i} \sum_{i=1}^{m} b_{i}+\sum_{i=1}^{m} a_{i}+\sum_{i=1}^{m} b_{i}-\frac{9 e}{32}\left(\sum_{i=1}^{m} a_{i}\right)^{2}-\frac{1}{2 e}\left(\sum_{i=1}^{m} b_{i}\right)^{2}, g \text { arbitrary. }
\end{array}\right.
$$

If $g$ is arbitrary, the claim follows since a thorough investigation leads to

$$
h^{0}\left(\Sigma, \mathcal{O}_{\Sigma}\left(\Delta_{i}\right)\right) \leq a_{i} b_{i}+a_{i}+b_{i}+1-\frac{9 e}{32} \cdot a_{i}^{2}
$$

and

$$
h^{0}\left(\Sigma, \mathcal{O}_{\Sigma}\left(\Delta_{i}\right)\right) \leq \frac{1}{4} \cdot a_{i} b_{i}+a_{i}+b_{i}+1-\frac{9 e}{32} \cdot a_{i}^{2}-\frac{1}{2 e} \cdot b_{i}{ }^{2} .
$$

If $g=1$, then $e=-1$ and $b=b^{\prime}+\frac{a}{2}$, and we are done since

$$
\begin{aligned}
h^{0}\left(\Sigma, \mathcal{O}_{\Sigma}\left(\Delta_{i}\right)\right) \leq a_{i} b_{i}^{\prime}+b_{i}^{\prime}+1 & +\frac{a_{i}\left(a_{i}+1\right)}{2}+\frac{b_{i}^{\prime}\left(b_{i}^{\prime}-1\right)}{2} \\
& =\frac{1}{2} \cdot a_{i} b_{i}+\frac{1}{2} \cdot b_{i}{ }^{2}+\frac{1}{8} \cdot a_{i}^{2}+\frac{1}{4} \cdot a_{i}+\frac{1}{2} \cdot b_{i}+1 .
\end{aligned}
$$

Step 5: In this last step we gather the information from the previous investigations and finish the proof considering a bunch of different cases.

Using Step 3 and Step 4 and taking $\sum_{i=1}^{m} a_{i}+b_{i} \leq m$ into account, we get the following upper bounds for $\beta^{\prime}=h^{1}\left(\Sigma, \mathcal{J}_{X_{0}}(D)\right)+\sum_{i=1}^{m}\left(h^{0}\left(\Sigma, \mathcal{O}_{\Sigma}\left(\Delta_{i}\right)\right)-1\right)$ :

$$
\beta^{\prime} \leq \begin{cases}3 \sum_{i=1}^{m} a_{i} \sum_{i=1}^{m} b_{i}+2 g \sum_{i=1}^{m} a_{i}, & \text { if } e=0, \\ 3 \sum_{i=1}^{m} a_{i} \sum_{i=1}^{m} b_{i}+2 g \sum_{i=1}^{m} a_{i}-\frac{9 e}{32}\left(\sum_{i=1}^{m} a_{i}\right)^{2}, & \text { if } e<0, \\ \frac{9}{4} \sum_{i=1}^{m} a_{i} \sum_{i=1}^{m} b_{i}+2 g \sum_{i=1}^{m} a_{i}-\frac{9 e}{32}\left(\sum_{i=1}^{m} a_{i}\right)^{2}-\frac{1}{2 e}\left(\sum_{i=1}^{m} b_{i}\right)^{2}, & \text { if } e<0, \\ 3 \sum_{i=1}^{m} a_{i} \sum_{i=1}^{m} b_{i}, & \text { if } e=0, g=1, \sum_{i=1}^{m} b_{i} \neq 0, \\ m \leq \sum_{i=1}^{m} a_{i}, & \text { if } e=0, g=1, \sum_{i=1}^{m} b_{i}=0, \\ \frac{5}{2} \sum_{i=1}^{m} a_{i} \sum_{i=1}^{m} b_{i}+\frac{1}{2}\left(\sum_{i=1}^{m} b_{i}\right)^{2}+\frac{1}{8}\left(\sum_{i=1}^{m} a_{i}\right)^{2}+\frac{5}{4} \sum_{i=1}^{m} a_{i}, & \text { if } e<0, g=1\end{cases}
$$


Applying now Steps $2 \mathrm{~b}-2 \mathrm{~d}$ we end up with $\frac{\beta^{\prime} \cdot\left(D-K_{\Sigma}\right)^{2}}{\left(\operatorname{deg}\left(X_{S}\right)\right)^{2}} \leq \gamma$. We thus finally get

$$
\begin{aligned}
& h^{1}\left(\Sigma, \mathcal{J}_{X_{0}}(D)\right)+\sum_{i=1}^{m}\left(h^{0}\left(\Sigma, \mathcal{O}_{\Sigma}\left(\Delta_{i}\right)\right)-1\right)=\beta^{\prime} \leq \frac{1}{\gamma \cdot\left(D-K_{\Sigma}\right)^{2}} \cdot\left(\operatorname{deg}\left(X_{S}\right)\right)^{2} \\
& \quad=\frac{1}{\gamma \cdot\left(D-K_{\Sigma}\right)^{2}} \cdot\left(\sum_{z \in \Sigma} \operatorname{deg}\left(X_{S, z}\right)\right)^{2} \leq \frac{\# X_{S}}{\gamma \cdot\left(D-K_{\Sigma}\right)^{2}} \cdot \sum_{z \in \Sigma} \operatorname{deg}\left(X_{S, z}\right)^{2} \\
& \quad \leq \frac{\# X_{S}}{\gamma \cdot\left(D-K_{\Sigma}\right)^{2}} \cdot \sum_{z \in \Sigma} \operatorname{deg}\left(X_{0, z}\right)^{2}<_{(4)} \# X_{S}
\end{aligned}
$$

It remains to show that the inequality that we derived cannot hold.

Lemma 4.7. Let $D \in \operatorname{Div}(\Sigma)$, let $\mathcal{S}_{1}, \ldots, \mathcal{S}_{r}$ be pairwise distinct topological or analytical singularity types and let $k_{1}, \ldots, k_{r} \in \mathbb{N} \backslash\{0\}$. Suppose that $V_{|D|}^{\text {irr,reg }}\left(k_{1} \mathcal{S}_{1}, \ldots\right.$, $\left.k_{r} \mathcal{S}_{r}\right)$ is nonempty.

Then there exists no curve $C \in V_{|D|}^{i r r}\left(k_{1} \mathcal{S}_{1}, \ldots, k_{r} \mathcal{S}_{r}\right) \backslash \overline{V_{|D|}^{\text {irr,reg }}\left(k_{1} \mathcal{S}_{1}, \ldots, k_{r} \mathcal{S}_{r}\right)}$ such that for the zero-dimensional scheme $X_{0}=X(C)$ there exist curves $\Delta_{1}, \ldots$, $\Delta_{m} \subset \Sigma$ and zero-dimensional locally complete intersections $X_{i}^{0} \subseteq X_{i-1}$ for $i=$ $1, \ldots, m$, where $X_{i}=X_{i-1}: \Delta_{i}$ for $i=1, \ldots, m$, such that $X_{S}=\bigcup_{i=1}^{m} X_{i}^{0}$ satisfies

$$
h^{1}\left(\Sigma, \mathcal{J}_{X_{0}}(D)\right)+\sum_{i=1}^{m}\left(h^{0}\left(\Sigma, \mathcal{O}_{\Sigma}\left(\Delta_{i}\right)\right)-1\right)<\# X_{S} .
$$

Proof. Throughout the proof we use the notation $V^{i r r}=V_{|D|}^{i r r}\left(k_{1} \mathcal{S}_{1}, \ldots, k_{r} \mathcal{S}_{r}\right)$ and $V^{\text {irr,reg }}=V_{|D|}^{\text {irr,reg }}\left(k_{1} \mathcal{S}_{1}, \ldots, k_{r} \mathcal{S}_{r}\right)$.

Suppose there exists a curve $C \in V^{i r r} \backslash \overline{V^{i r r, r e g}}$ satisfying the assumption of the lemma, and let $V^{*}$ be the irreducible component of $V^{i r r}$ containing $C$. Moreover, let $C_{0} \in V^{\text {irr,reg }}$.

In the following we consider the morphism from Subsection 1.5

$$
\Psi=\Psi_{|D|}\left(k_{1} \mathcal{S}_{1}, \ldots, k_{r} \mathcal{S}_{r}\right): V_{|D|}\left(k_{1} \mathcal{S}_{1}, \ldots, k_{r} \mathcal{S}_{r}\right) \rightarrow B\left(k_{1} \mathcal{S}_{1}, \ldots, k_{r} \mathcal{S}_{r}\right)=B .
$$

Step 1: $h^{0}\left(\Sigma, \mathcal{J}_{X\left(C_{0}\right) / \Sigma}(D)\right)=h^{0}\left(\Sigma, \mathcal{J}_{X(C) / \Sigma}(D)\right)-h^{1}\left(\Sigma, \mathcal{J}_{X(C) / \Sigma}(D)\right)$.

By the choice of $C_{0}$ we have

$$
0=H^{1}\left(\Sigma, \mathcal{J}_{X^{*}\left(C_{0}\right) / \Sigma}(D)\right) \rightarrow H^{1}\left(\Sigma, \mathcal{O}_{\Sigma}(D)\right) \rightarrow H^{1}\left(\Sigma, \mathcal{O}_{X^{*}\left(C_{0}\right)}(D)\right)=0,
$$

and thus $D$ is non-special, i.e., $h^{1}\left(\Sigma, \mathcal{O}_{\Sigma}(D)\right)=0$. But then

$$
h^{0}\left(\Sigma, \mathcal{J}_{X\left(C_{0}\right) / \Sigma}(D)\right)=h^{0}\left(\Sigma, \mathcal{J}_{X(C) / \Sigma}(D)\right)-h^{1}\left(\Sigma, \mathcal{J}_{X(C) / \Sigma}(D)\right) .
$$

Step 2: $h^{1}\left(\Sigma, \mathcal{J}_{X(C)}(D)\right) \geq \operatorname{codim}_{B}\left(\Psi\left(V^{*}\right)\right)$.

Suppose the contrary, that is, $\operatorname{dim}\left(\Psi\left(V^{*}\right)\right)<\operatorname{dim}(B)-h^{1}\left(\Sigma, \mathcal{J}_{X(C) / \Sigma}(D)\right)$. Then by Step 1 and Theorem 3.1

$$
\begin{aligned}
\operatorname{dim}\left(V^{*}\right) & \leq \operatorname{dim}\left(\Psi\left(V^{*}\right)\right)+\operatorname{dim}\left(\Psi^{-1}(\Psi(C))\right) \\
& <\operatorname{dim}(B)-h^{1}\left(\Sigma, \mathcal{J}_{X(C) / \Sigma}(D)\right)+h^{0}\left(\Sigma, \mathcal{J}_{X(C) / \Sigma}(D)\right)-1 \\
& =\operatorname{dim}(B)+h^{0}\left(\Sigma, \mathcal{J}_{X\left(C_{0}\right) / \Sigma}(D)\right)-1=\operatorname{dim}\left(V^{\text {irr }, \text { reg }}\right) .
\end{aligned}
$$

However, any irreducible component of $V^{i r r}$ has at least the expected dimension $\operatorname{dim}\left(V^{i r r, r e g}\right)$, which gives a contradiction. 
Step 3: $\operatorname{codim}_{B}\left(\Psi\left(V^{*}\right)\right) \geq \# X_{S}-\sum_{i=1}^{m} \operatorname{dim}\left|\Delta_{i}\right|_{l}$. The existence of the subschemes $X_{i}^{0} \subseteq X(C) \cap \Delta_{i}$ imposes at least $\# X_{i}^{0}-\operatorname{dim}\left|\Delta_{i}\right|_{l}$ conditions on $X(C)$ and increases thus the codimension of $\Psi\left(V^{*}\right)$ by this number.

Step 4: Collecting the results we derive the following contradiction:

$$
\begin{aligned}
h^{1}\left(\Sigma, \mathcal{J}_{X(C)}(D)\right) & \geq_{\text {Step 2 }} \quad \operatorname{codim}_{B}\left(\Psi\left(V^{*}\right)\right) \\
& \geq_{\text {Step 3 }} \quad \# X_{S}-\sum_{i=1}^{m} \operatorname{dim}\left|\Delta_{i}\right|_{l}>_{\text {(4.19) }} h^{1}\left(\Sigma, \mathcal{J}_{X(C)}(D)\right) .
\end{aligned}
$$

\section{REFERENCES}

[AC83] Enrico Arbarello and Maurizio Cornalba, A few remarks about the variety of irreducible plane curves of given degree and genus, Ann. Sci. École Norm. Sup. 16 (1983), no. 3, 467-488. MR 86a:14020

[Bar93] Didier Barkats, Irréductibilité des variétés des courbes planes à noeuds et à cusps, Preprint no. 364, Univ. de Nice-Sophia-Antipolis, 1993.

[Bog79] Fedor A. Bogomolov, Holomorphic tensors and vector bundles on projective manifolds, Math. USSR Izvestija 13 (1979), no. 3, 499-555. MR 80j:14014

[Bru99] Andrea Bruno, Limit linear series and families of equisingular plane curves, Preprint, 1999.

[CC99] Luca Chiantini and Ciro Ciliberto, On the Severi variety of surfaces in $\mathbb{P}_{\mathbb{C}}^{3}$, J. Algebraic Geom. 8 (1999), 67-83. MR 2000f:14082

[Che01] Xi Chen, Some remarks on the Severi varieties of surfaces in $\mathbb{P}^{3}$, Int. J. Math. Math. Sci. 26 (2001), no. 1, 1-5. MR 2002f:14011

[DH88] Steven Diaz and Joe Harris, Ideals associated to deformations of singular plane curves, Trans. Amer. Math. Soc. 309 (1988), 433-468. MR 89m:14003

[Fla01] Flaminio Flamini, Some results of regularity for Severi varieties of projective surfaces, Comm. Algebra 29 (2001), no. 6, 2297-2311. MR 2002c:14043

[GK89] Gert-Martin Greuel and Ulrich Karras, Families of varieties with prescribed singularities, Compositio Math. 69 (1989), 83-110. MR 90d:32037

[GL96] Gert-Martin Greuel and Christoph Lossen, Equianalytic and equisingular families of curves on surfaces, Manuscripta Math. 91 (1996), 323-342. MR 98g:14023

[GLS97] Gert-Martin Greuel, Christoph Lossen, and Eugenii Shustin, New asymptotics in the geometry of equisingular families of curves, Internat. Math. Res. Notices 13 (1997), 595-611. MR 98g:14039

[GLS98a] Gert-Martin Greuel, Christoph Lossen, and Eugenii Shustin, Geometry of families of nodal curves on the blown-up projective plane, Trans. Amer. Math. Soc. 350 (1998), 251-274. MR 98j:14034

[GLS98b] Gert-Martin Greuel, Christoph Lossen, and Eugenii Shustin, On the irreducibility of families of curves, Unpulished Manuscript, 1998.

[GLS98c] Gert-Martin Greuel, Christoph Lossen, and Eugenii Shustin, Plane curves of minimal degree with prescribed singularities, Inv. math. 133 (1998), 539-580. MR 99g:14035

[GLS00] Gert-Martin Greuel, Christoph Lossen, and Eugenii Shustin, Castelnuovo function, zero-dimensional schemes, and singular plane curves, J. Algebraic Geom. 9 (2000), no. 4, 663-710. MR 2001g:14045

[Har77] Robin Hartshorne, Algebraic geometry, Springer-Verlag, New York, 1977. MR 57:3116

[Har85] Joe Harris, On the Severi problem, Inventiones Math. 84 (1985), 445-461. MR 87f:14012

[Kan89a] Pyung-Lyun Kang, A note on the variety of plane curves with nodes and cusps, Proc. Amer. Math. Soc. 106 (1989), no. 2, 309-312. MR 89k:14046

[Kan89b] Pyung-Lyun Kang, On the variety of plane curves of degree $d$ with $\delta$ nodes and $k$ cusps, Trans. Amer. Math. Soc. 316 (1989), no. 1, 165-192. MR 90g:14014 
[KT02] Thomas Keilen and Ilya Tyomkin, Existence of curves with prescribed topological singularities, Trans. Amer. Math. Soc. 354 (2002), no. 5, 1837-1860, http://www. mathematik.uni-kl.de/ ${ }^{\sim}$ keilen/download/KeilenTyomkin001/KeilenTyomkin001.ps.gz. MR 2003a:14041

[Laz97] Robert Lazarsfeld, Lectures on linear series, Complex Algebraic Geometry (János Kollár, ed.), IAS/Park City Mathematics Series, no. 3, Amer. Math. Soc., Providence, RI, 1997, pp. 161-219. MR 98h:14008

[Los98] Christoph Lossen, The geometry of equisingular and equianalytic families of curves on a surface, Ph.D. Thesis, FB Mathematik, Universität Kaiserslautern, Aug. 1998, http://www.mathematik.uni-kl.de/ ${ }^{\prime}$ lossen/download/Lossen002/Lossen002.ps.gz.

[Mil68] John Milnor, Singular points of complex hypersurfaces, Princeton University Press, Princeton, NJ, 1968. MR 39:969

[Ran89] Ziv Ran, Families of plane curves and their limits: Enriques' conjecture and beyond, Annals of Math. 130 (1989), no. 1, 121-157. MR 90e:14024

[Sev21] Francesco Severi, Vorlesungen über Algebraische Geometrie, Bibliotheca Mathematica Teubneriana, no. 32, Teubner, 1921.

[Shu91a] Eugenii Shustin, Geometry of discriminant and topology of algebraic curves, Proc. Internat. Congress Math., Kyoto 1990 (Tokyo, Berlin, New York), vol. 1, SpringerVerlag, 1991. MR 93h:14018

[Shu91b] Eugenii Shustin, On manifolds of singular algebraic curves, Selecta Math. Sov. 10 (1991), 27-37. MR 91k:00045

[Shu94] Eugenii Shustin, Smoothness and irreducibility of varieties of plane curves with nodes and cusps, Bull. Soc. Math. France 122 (1994), 235-253. MR 95e:14020

[Shu96a] Eugenii Shustin, Geometry of equisingular families of plane algebraic curves, J. Alg. Geom. 5 (1996), 209-234. MR 97g:14025

[Shu96b] Eugenii Shustin, Smoothness and irreducibility of varieties of algebraic curves with ordinary singularities, Israel Math. Conf. Proc., no. 9, Amer. Math. Soc., 1996, pp. 393416. MR 97c: 14028

[Wal96] Charles T.C̃. Wall, Highly singular quintic curves, Math. Proc. Cambridge Philos. Soc. 119 (1996), 257-277. MR 97b:14058

[Zar35] Oscar Zariski, Algebraic surfaces, vol. III, Ergebnisse der Mathematik und ihrer Grenzgebiete, no. 5, Springer, 1935. MR 57:9695

Universität Kaiserslautern, Fachbereich Mathematik, Erwin-Schrödinger-Strasse, D-67663 Kaiserslautern, Germany

E-mail address: keilen@mathematik.uni-kl.de

$U R L:$ http://www. mathematik. uni-kl.de/ keilen 